\title{
Optimization Techniques for Improving the Performance of Silicone-Based Dielectric Elastomers
}

Skov, Anne Ladegaard; Yu, Liyun

Published in:

Advanced Engineering Materials

Link to article, DOI:

10.1002/adem.201700762

Publication date:

2018

Document Version

Early version, also known as pre-print

Link back to DTU Orbit

Citation (APA):

Skov, A. L., \& Yu, L. (2018). Optimization Techniques for Improving the Performance of Silicone-Based Dielectric Elastomers. Advanced Engineering Materials, 20(5), [1700762].

https://doi.org/10.1002/adem.201700762

\section{General rights}

Copyright and moral rights for the publications made accessible in the public portal are retained by the authors and/or other copyright owners and it is a condition of accessing publications that users recognise and abide by the legal requirements associated with these rights.

- Users may download and print one copy of any publication from the public portal for the purpose of private study or research.

- You may not further distribute the material or use it for any profit-making activity or commercial gain

- You may freely distribute the URL identifying the publication in the public portal 
Optimization techniques for improving the performance of silicone-based dielectric elastomers

Anne Ladegaard Skov* and Liyun Yu

Danish Polymer Centre, Department of Chemical Engineering, DTU, Denmark

*al@kt.dtu.dk 


\title{
Optimization techniques for improving the performance of silicone-based dielectric elastomers
}

\author{
Dielectric elastomers are possible candidates for realizing products that are in high \\ demand by society, such as soft robotics and prosthetics, tactile displays, and smart \\ wearables. Diverse and advanced products based on dielectric elastomers are available; \\ however, no elastomer has proven ideal for all types of products. Silicone elastomers, \\ though, are the most promising type of elastomer when viewed from a reliability \\ perspective, since in normal conditions they do not undergo any chemical degradation \\ or mechanical ageing/relaxation. Within this review, different pathways to improving \\ the electro-mechanical performance of dielectric elastomers are highlighted. Various \\ optimization methods for improved energy transduction are investigated and discussed, \\ with special emphasis placed on the promise each method holds. The compositing and \\ blending of elastomers are shown to be simple, versatile methods that can solve a \\ number of optimization issues. More complicated methods, involving chemical \\ modification of the silicone backbone as well as controlling the network structure for \\ improved mechanical properties, are shown to solve yet more issues. From the analysis, \\ it is obvious that there is not one single optimization technique that will lead to the \\ universal optimization of dielectric elastomer films, though each method may lead to \\ elastomers with certain features and thus certain potentials.
}

Keywords: dielectric elastomers, smart materials, silicone, optimization, mechanical testing

\section{Introduction}

Dielectric elastomers hold great promise as materials for novel, advanced electromechanical applications such as actuators, generators, and sensors, due to their simple, linear, and flexible working principle combined with the promise of lightweight and cheap transducers[1-7]. Dielectric elastomers consist of an elastomer film placed between two thin and compliant electrodes, thereby creating a capacitor capable of energy transduction, as shown in Figure 1. The electrodes can be prepared from a wide selection of conducting 
materials, such as noble metals[8,9] and carbon black-polymer mixtures[10-12]. For a detailed and thorough review on electrode systems, see Rosset and Shea[13] as well as the more recent review by McCoul et al.[14] In the following, the elastomer and electrode system is referred to as a "dielectric elastomer transducer" (DET), whereas the elastomer material will be denoted as "dielectric elastomer".
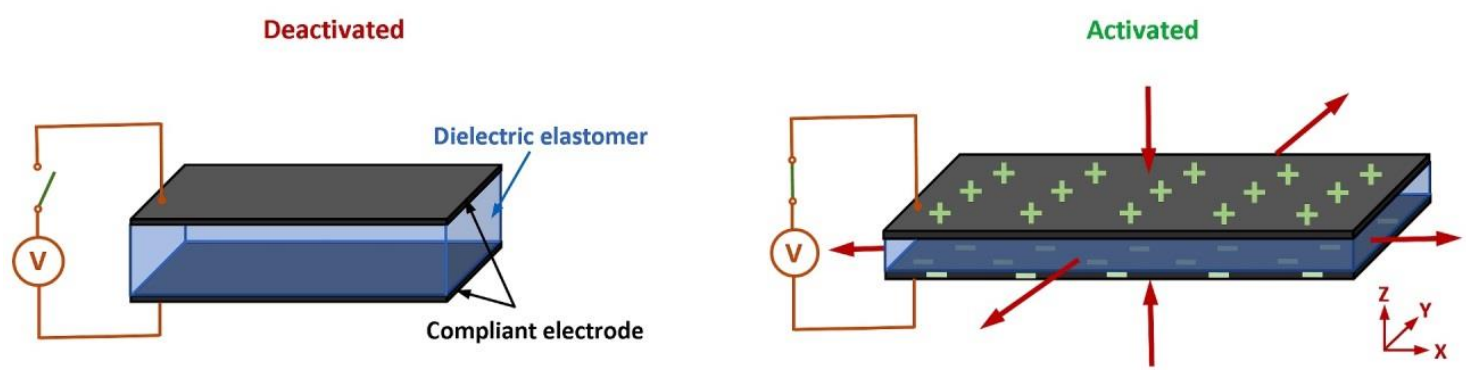

Figure 1: Illustration of the actuation principle behind dielectric elastomer transducers (DETs), which consist of a dielectric elastomer film and thin, compliant electrodes coupled to an external voltage supply. Sensing involves reading out differences in capacitance when stretching the transducer. Energy generation involves stretching the electrodes and applying a charge to the electrodes, before releasing external stress. Upon the release of stress, energy density increases.

When applying an external voltage to electrodes, electrostatic forces are created. Actuation has two sources of origin in the case of flexible electrodes, but for both flexible and rigid electrodes, first of all electrostatic pressure squeezes the elastomer perpendicular to the electrodes (i.e. in thickness). For flexible electrodes, yet another driving force is the increased charge repulsion of similar charges, leading to double electrostatic force when using compliant electrodes. Silicone elastomers can be regarded as incompressible, since they usually have a Poisson ratio of $0.49[15]$ or closer to 0.5 , which is the value for completely incompressible material. Consequently, the elastomer expands in the electrode plane (area), if not mechanically prohibited to one in-plane direction[16,17]. Electrical energy, in the 
illustrated case, is therefore converted into mechanical energy by so-called "linear motion"[18] as opposed to rotational motion for most other types of transducer. The physical incentive for the actuation process is reduced charge density when enlarging the electrode area and decreasing thickness. When the external voltage is switched off, the elastomer film ideally returns to its original shape[19].

The same principle can be used for the dielectric elastomer to be utilized as a sensor, i.e. deformation leads to a change in capacitance, and thus a capacitance reading can be coupled to deformation. Sensors based on dielectric elastomers are already widely available, such as those produced by StretchSense[20]. For sensors, most commercial silicone elastomers have very good performance, and therefore no further material optimization of the dielectric elastomer is needed. Better electrodes, however, are still in high demand, to allow for even further sensing capabilities[13].

Dielectric elastomers also find use as generators in creating electrical energy from mechanical energy. When stretching the DET via an external force, and the subsequent application of electrical charges onto the film, followed by the release of an external force, the DET immediately relaxes into an equilibrium state where electrical stress equals mechanical stress[21,22]. During stress equilibration, opposite charges on the two electrodes are forced further apart because the film increases in thickness, while similar charges to the respective electrodes are concentrated due to a decrease in the electrode area, thus increasing energy density. These charge density changes increase the voltage, so generated electrical energy can be collected[23].

For both actuators and generators, a common technique employed to improve performance involves formulating high-permittivity elastomers by means of physically or chemically including high permittivity fillers and moieties. Either of these modifications affects overall silicone elastomers, but there exist no guidelines on designing dielectric 
elastomers for specific applications, and it may be difficult to figure out which technique should be used for specific applications. In this work, we discuss various techniques based on simple scaling laws in terms of favorable properties as well as limitations.

\subsection{Fundamental equations and figures of merit}

Usually, the actuation equation by Pelrine et al.[24] is used as a starting point for optimization:

$$
S_{\max }=\frac{\epsilon_{0} \epsilon_{r}}{Y}\left(\frac{V}{d}\right)^{2}
$$

where $\epsilon_{0}$ is vacuum permittivity, $\epsilon_{r}$ is dielectric permittivity, $Y$ is the Young's modulus, $V$ is voltage, and $d$ is film thickness. This equation is a very simplified equation and ignores material non-linearities and pull-in effects[25], though it is still a useful approximation. Silicone elastomers are usually rather linear in their stress-strain response, except that they sometimes have a large initial Young's modulus for strains of approximately $0-2 \%$, as discussed later in the context of the Mullins and the Payne effects. Many other soft elastomers, such as the acrylics-based adhesive VHB, however, are very non-linear in their responses[26], and the actuation equation covers only a very limited range of strains.

In terms of ultimate actuation strains achievable, Equation 1 can be written as:

$$
S_{\max }=\frac{\epsilon_{0} \epsilon_{r}}{Y} E_{B}^{2}
$$

where $E_{B}$ is electrical breakdown strength, i.e. the maximum applicable voltage to a film of a given initial thickness. Here it is assumed that the first breakdown that takes place is electrical in nature. This is a relatively reasonable assumption for silicones, which usually have ultimate strains far beyond 300\%. However, electromechanical instability (EMI) is ignored as a possible breakdown mechanism in the expression. This will be discussed later. 
This equation was modified in the work of Sommer-Larsen and Larsen[27] to a figure of merit, namely that the figure of merit for a given dielectric elastomer utilized as an actuator can be determined from:

$$
F_{\text {om }}^{\text {traditional }}(D E A)=\frac{3 \epsilon_{r} \epsilon_{0} E_{B}^{2}}{Y}
$$

From this equation, it is obvious that with respect to the elastomer material, three material properties directly affect the actuation properties of dielectric elastomers, namely $\epsilon_{r}$, $Y$, and $E_{B}$. The figure of merit in Equation 3 includes electrical breakdown strength, but in many instances a given voltage will be applicable to a given product, such as a particular battery. Therefore, in this work, we will use a simpler figure of merit for actuators, i.e. one that reveals actuation strain improvements for a certain applied electrical field below the electrical breakdown strength (or the EMI) of the given elastomer. This figure of merit is given by:

$$
F_{o m}(D E A)=\frac{\epsilon_{r}}{Y} / \frac{\epsilon_{r, 0}}{Y_{0}}=\frac{\epsilon_{r}}{\epsilon_{r, 0}} \frac{Y_{0}}{Y}
$$

where subscript 0 denotes reference material values, and the absence of a subscript denotes investigated material values.

However, other material parameters, as well as processability of the developed elastomers, are also important for achieving reliable actuation and will be discussed in the context of each modification technique.

Again, almost the same parameters appear in the figure of merit expression for generators, as derived by McKay et al.[28]:

$$
F_{\text {om }}(D E G)=\frac{\epsilon_{r} \epsilon_{0} E_{B}^{2}}{2 \varphi}
$$

where $\varphi$ is the strain energy function of the elastomer.

As discussed in a recent review, by Madsen et al.[29], of experimental results achieved by modifying silicone elastomers, several methodologies were proposed to increase 
the energy density, mainly via targeting increased dielectric permittivity. Broadly categorized, they fall under the following categories: 1) Silicone composites, 2) silicone/polymer blends, 3) chemically modified silicones, and 4) systems with a complex network structure. These techniques will be discussed in detail in subsequent sections, following a discussion of the curing mechanisms of silicone elastomers and their effects on formulation strategies.

\subsection{Curing chemistries of silicone elastomers}

In the previous review, reported experimental results were summarized for various silicone elastomer systems, based mainly on addition or condensation curing elastomers, as illustrated in Scheme 1. Addition curing elastomers are by far the easiest to formulate and prepare in a reliable way, due to the absence of side reactions and the almost full conversion of the socalled "silylation reaction"[30]. However, for many permittivity enhancement methods, the poisoning of the Pt catalyst poses a great challenge. Furthermore, nitrogen, sulphur, and aromatic components may all inhibit curing, either fully or to some extent, and thus they may lead to poorly crosslinked silicone elastomers. This was the case, for example, for one of the first reported silicone-based systems, with grafted dipoles inhibiting the addition curing elastomer. However, significant actuation was achieved through a combination of an increase in permittivity and the inherent softness of the incomplete network[31], albeit these elastomers were not reliable over time. 
<smiles>[R]O[R]OS(C)(C)O[R]</smiles>

PDMS

hydride crosslinker

addition cured silicone

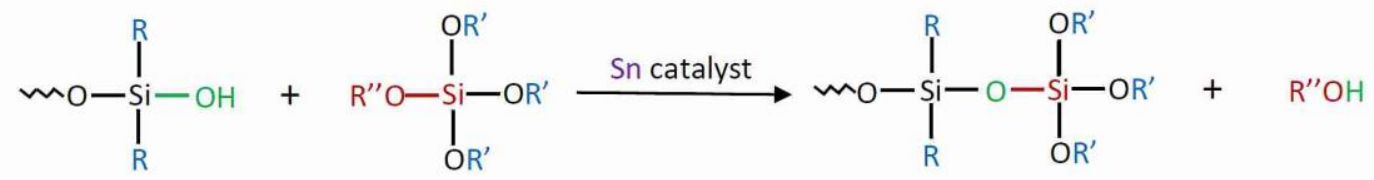

PDMS

silicic acid crosslinker

condensation cured silicone

water or alcohol

$R, R^{\prime}=$ various groups such as $\mathrm{CH}_{3}$, phenyl or polymer chain

$R^{\prime \prime}=$ hydrogen or alkyl

Scheme 1: Addition curing (also known as silylation) and condensation curing of silicone polymers into silicone elastomers. The illustration shows one reaction step only of a series of chemically identical reactions leading to crosslinking.

In the present article, each of these optimized elastomer systems will be discussed in detail, not only with respect to potential actuation improvement, but also with respect to reliability. The curing mechanism behind turning silicone polymers into silicone elastomers is not regarded as a tuning parameter, since the elastomers from both curing types possess similar properties. However, comments on situations where a certain curing mechanism is required will be added, where relevant. Potentials are derived by using simple scaling laws as well as rules of thumb developed over several years of extensive silicone elastomer formulation experience. The results should be directly applicable to other elastomer systems such as polyurethane-based and acrylic-based elastomers, since the orders of magnitude of improvement should be similar for all soft elastomer systems, independent of the chemistry of the elastomer matrix. One main difference, however, is that most polyurethane- and acrylate-based elastomers are not filled with particulate fillers in the same way as silicone 
analogous varieties, due to the stronger inter-chain interactions of these polymers, and thus there is less need for reinforcement.

\section{Traditional composite systems}

Commercially available silicone elastomers usually contain significant amounts of fillers. The traditional choice of fillers for silicones is silica particles, due to their inherent compatibility. Moreover, as a result of strong particle-particle and particle-network interactions, these particles provide improved ultimate properties for the otherwise relatively weak silicone network, which is dominated by weak inter-chain van der Waals forces alongside covalent crosslinking points[32]. Silica, however, does not contribute positively to the dielectric permittivity of the resulting elastomer, since the relative permittivities of silica and silicone are similar[33]. Therefore, high-permittivity fillers such as metal oxide fillers are required in order to meet the demand for enhanced dielectric elastomer energy density. These fillers can either — via the simplest process — be blended directly into one or both of the elastomer premixes[33-42] or alternatively_via the more demanding process—incorporated through in-situ condensation reactions during the crosslinking condensation reaction[43,44], thereby—in both cases_-yielding a morphology as illustrated in Figure 2.

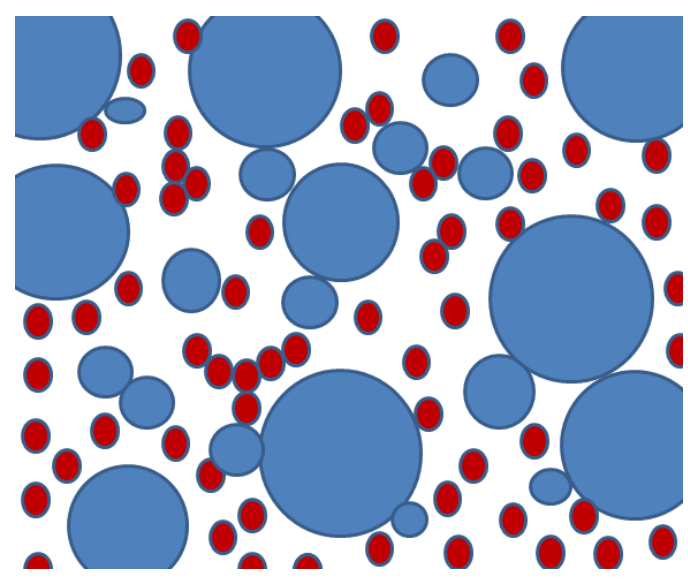

Figure 2: Illustration of a cross-section of a silicone elastomer composite with two types of particulate fillers, namely silica (small red spheres) and metal oxide particles (large blue 
spheres). Silica particles are usually on the nanometer scale, whereas metal oxides are on the large nanometer or small micrometer scale (roughly 100-2000 nm).

With the introduction of nano-sized particulate fillers, the resulting mechanical properties of the elastomer composite will change significantly. The elastomer composite will have at least two types of particle interfaces within the matrix, namely those between the reinforcing silica and the permittivity-enhancing metal oxide, respectively, with the silicone matrix. Silica-metal oxide interactions may also contribute strongly to elastomer properties, while metal oxide-metal oxide interactions can also alter mechanical properties. Obviously, the complexity of the elastomer increases in line with the introduction of yet another component, and the Mullins effect is supposed to become inevitable. The Mullins effect causes stress softening of the elastomer after the first deformation, as illustrated in Figure 3. The effect is generally assumed to result from a change in the microstructure, wherein the effective volume fraction of the hard domain decreases with stretching, from irreversible damage to the material, or from a combination of both aspects[45,46]. Practically, the Mullins effect leads to differences between the first actuation cycle and the following cycles, or alternatively, if introduced during processing, the resulting dielectric elastomer films may appear with mechanical properties, depending on the maximum mechanical stretching experienced during processing. Some pristine commercial elastomers such as Elastosil RT625 show a very limited Mullins effect (less than $0.5 \%$ strain after cycling to $120 \%$ strain[47]); however, many formulated silicone composites do show significant Mullins effects, and therefore the design of any given product has to consider this issue. 

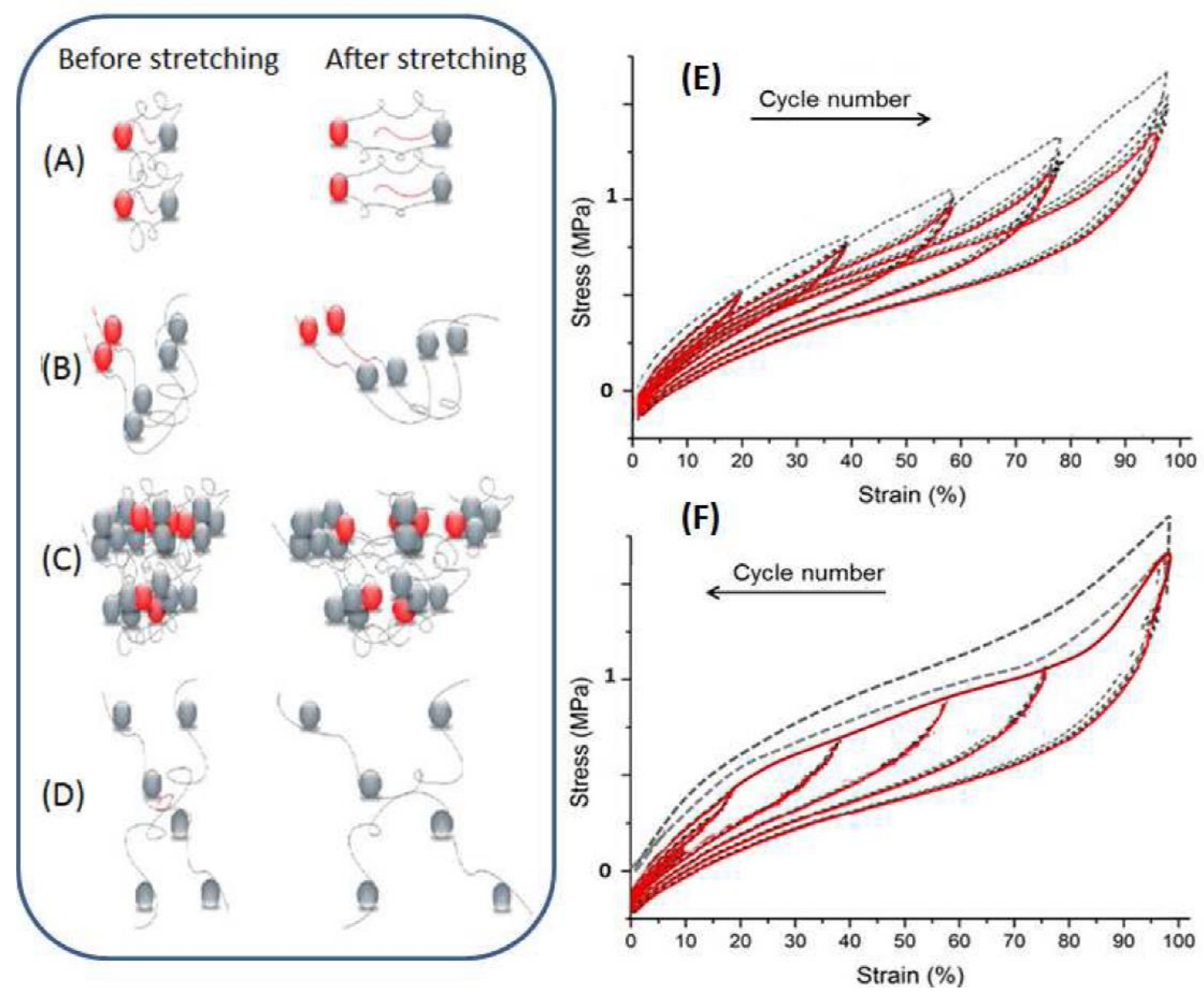

Figure 3: Four phenomena used to explain the Mullins effect. (A) Bond rupture of covalent bonds in a polymer chain, (B) slipping of molecules across particles, (C) filler rupture (aggregates break up), and (D) disentanglement of polymer chains. Stress-strain behavior for elastomers undergoing the Mullins effect: (E) Illustrates cycles with increasing strain, and (F) illustrates cycles with decreasing strain, i.e. only one occurrence of the Mullins effect, namely at the initial, highest applied strain amplitude (the only two fully gray curves). The red curves indicate the stable stress-strain performance obtained after approximately three cycles.

Yet another phenomenon influencing the mechanical performance and modeling of filled elastomers is the Payne effect, which is a particular feature of the stress-strain behavior of filled elastomers or rubber, especially highly crosslinked elastomer (rubber) compounds 
with fillers with aspect ratios close to 1, i.e. spherical fillers[48]. The Payne effect - in the same way as for the Mullins effect—is detected under cyclic loading conditions, albeit at small strain amplitudes, in contrast to the Mullins effect. Furthermore, the Payne effect is manifested through the strain amplitude dependency of the viscoelastic storage modulus $\left(\mathrm{G}^{\prime}\right)$. Above approximately $1 \%$ strain, the storage modulus is observed to decrease rapidly in line with increasing strain. At larger strains (usually higher than 10\%, which confusingly, as a rule of thumb, is also the upper value for the linear regime for soft silicone elastomers), the storage modulus approaches a lower plateau, which is illustrated in Figure 4. This smallstrain dependency may complicate the exact definition of the linear viscoelastic region (a region where the shear modulus for the given material is independent of strain), since these elastomers/rubbers commonly have two linear viscoelastic regions (one at intermediate strains and another one at low strains). This is also the reason for commonly encountered discrepancies in the reporting of storage moduli, especially due to the coinciding values discussed above.

(A)

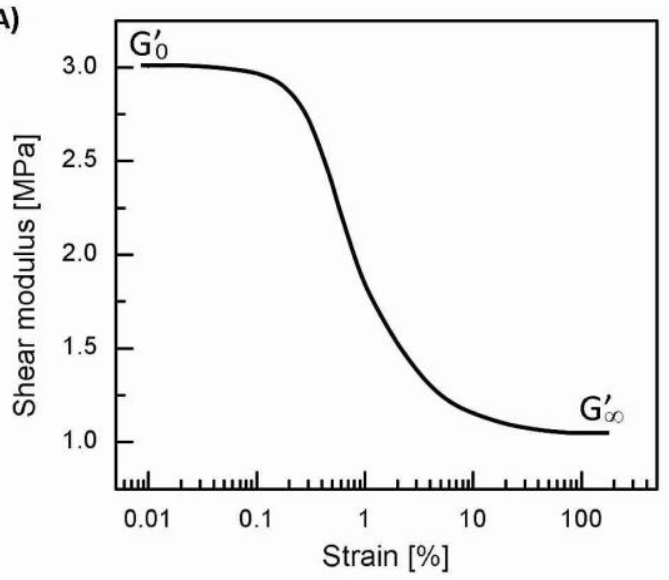

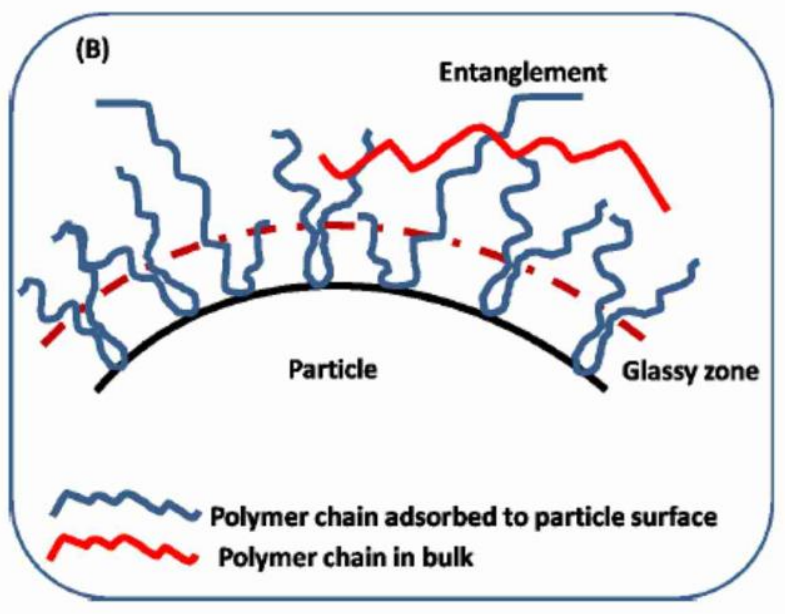

Figure 4: The Payne effect. (A) An elastomer showing the clear distinction of Payne effect softening for strain-sweeps performed at a constant frequency, usually $1 \mathrm{~Hz}$. The high-strain plateau in the shear modulus $\mathrm{G}_{\infty}{ }_{\infty}$ is the elastic contribution arising from the polymeric network, i.e. the sole contribution for unfilled elastomers. $\mathrm{G}_{0}{ }_{0}$ is the shear modulus at the 
given frequency. (B) The glassy zone, which is the interfacial zone between the particle surface and the bulk polymer network. The glass-like structure arises due to the local structuring and ordering of the polymer chains.

The Payne effect depends on the filler content and the aspect ratio of fillers[49,50]. Unfilled elastomers do not show this effect, since the sole contribution of such elastomers to elasticity arises from the polymer. For most of the commercial silicone elastomers investigated within our laboratories, the Payne reduction is of the order of a factor 3, which means that approximately $75 \%$ of the elasticity in common dielectric elastomer formulations arises from particle interactions of various kinds. Physically, the Payne effect can be ascribed to deformation-induced changes in the interactions, in this case mainly particle-particle interactions. These changes include the breakage and recovery of physical bonds linking adjacent filler clusters, and the Payne effect is usually observed as differences in shear moduli at low and high strains. The Payne effect can explain the initial rigidity of some silicone elastomer composites, due to their initial Young's modulus being very high and opposing actuation, though the material softens above approximately $1 \%$ strain and complies again with the electrical field. The initial rigidity of the elastomer is due to strong particleparticle interactions that are broken down during the initial deformation.

\subsection{Empirical descriptions of composites' figures of merit}

In order to evaluate the feasibility of the compositing methodology[51,52], scaling relations of various constants are derived. Predicting the Young's modulus of the resulting composite is not trivial, but for simplicity, it is assumed that it scales with the volume fraction of the filler via a linear mixing rule, commonly utilized as the upper limit for large-particle composites[53]: 


$$
Y=Y_{0}\left(1-\Phi_{\text {filler }}\right)+\Phi Y_{\text {filler }} \Phi_{\text {filler }}=Y_{0}\left(1-\left(1-k_{Y}\right) \Phi_{\text {filler }}\right)
$$

where $\Phi_{\text {filler }}$ is the volume fraction of filler related to the volume fraction of the network by $\Phi_{\text {network }}+\Phi_{\text {filler }}=1$. The constant $k_{Y}=\Phi Y_{\text {filler }} / Y_{0}$ accounts for the moduli of filler $\left(Y_{\text {filler }}\right)$ and the unfilled elastomer $\left(Y_{0}\right)$ as well as an orientational parameter $\Phi=[0-1]$ describing the dispersion of fillers[53]. Due to differences in Young's moduli between the fillers and silicone, usually orders of magnitude, $k_{Y} \gg 1$, and thus, Equation 6 reduces to:

$$
Y \simeq Y_{0}\left(1+k_{Y} \Phi_{\text {filler }}\right)
$$

The improvement in dielectric permittivity is likewise assumed to be described by a linear mixing rule:

$$
\epsilon_{r}=\epsilon_{r, 0}\left(1-\left(1-k_{\epsilon}\right) \Phi_{\text {filler }}\right) \simeq \epsilon_{r, 0}\left(1+k_{\epsilon} \Phi_{\text {filler }}\right)
$$

where $k_{\epsilon}=\epsilon_{r, \text { filler }} / \epsilon_{r, 0}$ and $k_{\epsilon} \gg 1$ for the compositing methodology, in order to provide useful elastomer properties.

Thus, the improved energy density for a given electrical field below the electrical breakdown field of the elastomer composite can be described by:

$$
\frac{\epsilon}{Y} / \frac{\epsilon_{0}}{Y_{0}}=\frac{1+k_{\epsilon} \Phi_{\text {filler }}}{1+k_{Y} \Phi_{\text {filler }}}
$$

This relation is plotted in Figure 5 for various realistic combinations of $k_{\epsilon}$ and $k_{Y}$. Where $k_{\epsilon}=1000$ and $k_{Y}=2000$, this represents a typically high-permittivity metal oxide blended into a silicone elastomer with reasonable interactions between the metal oxide and the silicone elastomer, and thus a large $k_{Y}=\Phi Y_{\text {filler }} / Y_{0}$ value. Where $k_{\epsilon}=1000$ and $k_{Y}=100$, this represents the same situation as above, albeit the interaction between metal oxide and silicone elastomer is vanishing $(\Phi \rightarrow 0)$. This situation indicates very poor compatibility between the filler and the elastomer, and from a reliability point of view, this is not favorable, since phase separation over time is likely because there are no significant interfacial forces to keep the particles apart. This means that with large strains, the composite 
may rearrange into a more thermodynamically favorable morphology when the filler particles move into close proximity and aggregate. $k_{\epsilon}=100$ and $k_{Y}=50$ is used to illustrate a relatively softer but compatible filler with good permittivity. These numbers represent well the various systems of titanates ( $\mathrm{TiSr}, \mathrm{CuCaTi}$ and $\mathrm{TiBa}$ oxides) with surface modifications[54].

From Equation 9, it is obvious that if a significant actuation improvement at a given voltage is desired, then the following relation needs to hold: $k_{\epsilon} \gg k_{\Phi}$. In other words, the ratio of permittivities should be significantly larger than the ratio of Young's moduli multiplied by constant accounting for the spatial incorporation of filler. Unfortunately, though, this requirement is not fulfilled too often for filler-elastomer combinations. As an example, barium titanate $\left(\mathrm{BaTiO}_{3}\right)$ has a Young's modulus of $Y_{\text {filler }}=70 \mathrm{GPa}$ and a relative dielectric permittivity of $\epsilon_{r, \text { filler }}=5000$. If blended into a common silicone elastomer with $\epsilon_{r, 0}=2.5$ and $Y_{0}=1 \mathrm{MPa}$, the following constants are obtained: $k_{\epsilon}=2,000$ and $k_{Y}=$ $70,000 \Phi$. Therefore, $\Phi$ needs to be smaller than $2 / 70$, if any improvement is to be achieved. This value, however, indicates that a given particulate filler does not improve the mechanical properties of the composite, i.e. mechanical integrity most likely is lost due to a lack of strong interfacial forces between particles and the elastomer matrix (or stronger interactions). Thus, there is no achievable improvement to the dielectric elastomer actuator for stable and reliable elastomers with mechanical integrity ( $\Phi>0.1$ as a rule of thumb), if judged solely on energy density; rather, a substantial decrease in actuation performance is experienced.

As a final comment on the dielectric elastomer from compositing to be utilized as actuators, it must be emphasized that low concentrations of fillers may still work in some formulations. A recent example is the system of incompatible $\mathrm{ZnO}$ in commercial silicone elastomers, where it is evident that some commercial elastomers are capable of compatibilizing small concentrations of this filler, most likely due to certain additives in the 
given elastomers[55]. However, for such formulations, it has also been shown that unreliable elastomers appear above a few phr of $\mathrm{ZnO}$. The phr (parts per hundred rubber) unit is often used due to its simplicity when formulating, since it states the weight of a given substance per $100 \mathrm{~g}$ of rubber. At low filler loadings, phr resembles the weight percentage of filler. For this system, particulate filler is used as a voltage stabilizer for the elastomer, as will be discussed in more detail in the section on the chemical modification of silicone elastomers.

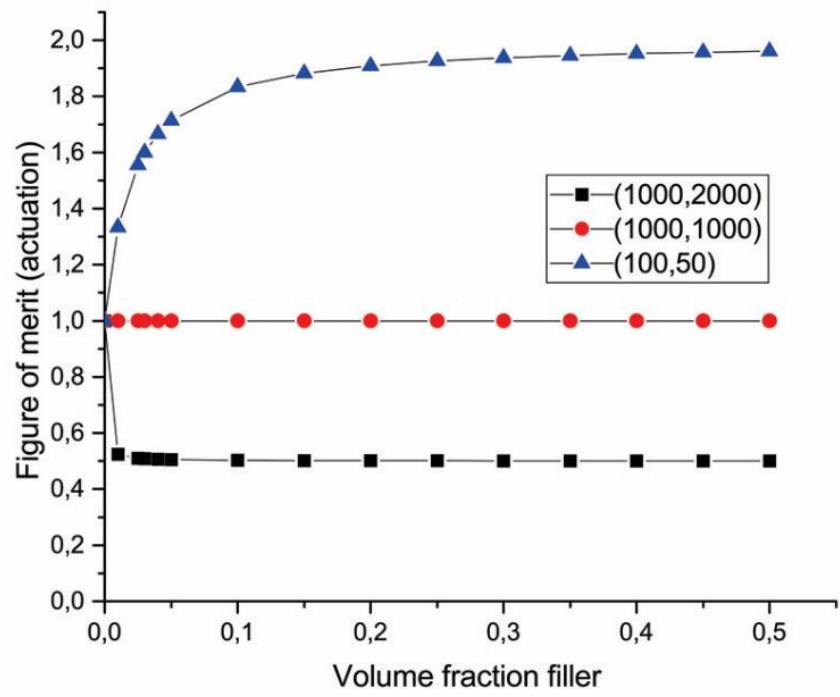

Figure 5: Figure of merit in actuator mode for composite elastomers with varying ratios of the Young's moduli and permittivities $\left(k_{Y}, k_{\epsilon}\right)$ stated in the legend.

However, for dielectric elastomers utilized as generators, the Young's modulus should preferably be large, since the forces involved are in general large, and thus mechanical resistance is not necessarily unfavorable. This requirement is fulfilled by composite elastomers, and in the following, an evaluation of the performance of composites as generators is derived, for which knowledge on electrical breakdown strength at a given filler loading is required, in order to know the operational maximum electrical field for energy generation. 
Non-conductive fillers have been shown to increase electrical breakdown strength significantly, for example as discussed in Yu and Skov[56], in which a linear relation between electrical breakdown strength and the Young's modulus was found:

$$
E_{B D}=E_{B D, 0}+k_{B D}\left(Y-Y_{0}\right)
$$

where $k_{B D}$ is the proportionality constant between electrical breakdown strength and Young's modulus. $E_{B D}$ and $E_{B D, 0}$ are the electrical breakdowns for the reference elastomer with an Young's modulus of 1MPa. Equation 10 is made dimensionless and the following expression is obtained:

$$
E_{B D}=E_{B D, 0}\left(1+\frac{k_{B D} Y_{0}}{E_{B D, 0}}\left(\frac{Y}{Y_{0}}-1\right)\right)=E_{B D, 0}\left(1+k_{B D}^{\star}\left(\frac{Y}{Y_{0}}-1\right)\right)
$$

where $k_{B D}^{\star}$ is the modified, dimensionless proportionality factor between $E_{B D}$ and the normalized Young's modulus $\left(Y-Y_{0}\right) / Y_{0}$ given by $k_{B D}^{\star}=k_{B D} Y_{0} / E_{B D, 0}$. In other words, a positive $k_{B D}^{\star}$ value indicates that adding a given filler increases electrical breakdown strength, which is the case for most metal oxides. Due to the normalized nature of $k_{B D}^{\star}$, a value of $k_{B D}^{\star}=0.4$, for example, means that if the Young's modulus is doubled, then the electrical breakdown strength will increase by $40 \%$.

Combining the expression in Equation 11 with Equation 7 results in the following relation:

$$
E_{B D}=E_{B D, 0}\left(1+k_{Y} k_{B D}^{\star} \Phi_{\text {filler }}\right)
$$

By combining Equations 7, 8, and 11, the following relation is obtained:

$$
\frac{\epsilon_{r} E_{B D}^{2}}{\epsilon_{r, 0} E_{B D, 0}^{2}} \simeq\left(1+k_{\epsilon} \Phi_{\text {filler }}\right)\left(1+k_{B D}^{\star} k_{Y} \Phi_{\text {filler }}\right)^{2}
$$

The equation is plotted in Figure 6 for various realistic combinations of the three parameters. From this description, it is obvious that composite-type dielectric elastomers hold great promise as generators. However, it is important to keep in mind that $\Phi_{\text {filler }}$ cannot be 
increased in perpetuity without causing other problems, such as ageing and loss of tension over time[47].

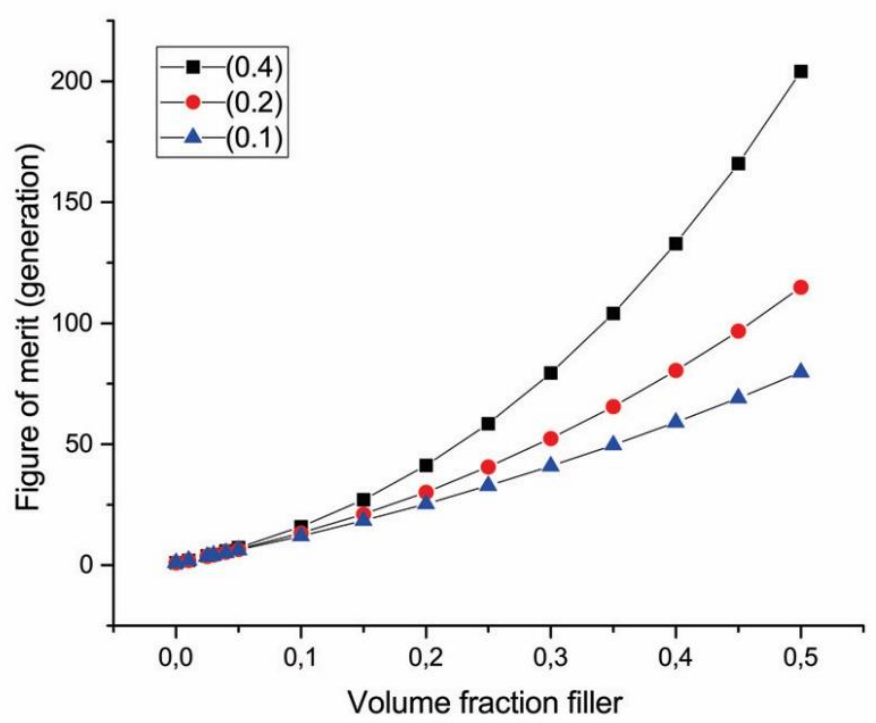

Figure 6: Figure of merit in generator mode for composites with varying values for $k_{B D}^{\star}$ (as defined in Equation 12) stated in the legend for constant values of $k_{\epsilon}=100$ and $k_{Y}=10$. $k_{B D}^{\star}$ states the increase in electrical breakdown strength when the Young's modulus is doubled, illustrated herein through $10 \%, 20 \%$, and $40 \%$ increases in electrical breakdown strength.

From Yu and Skov[56], $k_{B D}$ was determined to be $\sim 40(\mathrm{~V} / \mu \mathrm{m}) / \mathrm{MPa}$ from a plot of electrical breakdown strength as a function of the Young's modulus for various silicone elastomers with titanium dioxide fillers of various kinds (particle size, surface functionalization, etc.). The given $k_{B D}$ provides a $k_{B D}^{\star}$ value of approximately 0.4 , since $\mathrm{Y}=1$ $\mathrm{MPa}$ and $E_{B D, 0}=100 \mathrm{~V} / \mu \mathrm{m}$ as a guiding value for intermediate-strength silicone elastomers. This value of $k_{B D}$ is a good rule of thumb when changing elastomer formulations with nonconductive particulate fillers such as most of the metal oxides and silica. Much stronger dependencies of electrical breakdown strength on the Young's modulus have been 
found[57,58], but as mentioned, it depends on the mechanical integrity of the composite and on the electrical properties of the fillers, in which case no universal behavior is expected. Furthermore, the water content of the resulting elastomer composite also plays a significant role, especially for elastomers crosslinked by condensation chemistry with water as a byproduct. Stronger dependencies of electrical breakdown strength on the Young's modulus will favor even further composite elastomers with high loadings of filler for generator applications. An obvious example of a silicone elastomer where the simple, conservative rule of thumb does not hold is the new Wacker Elastosil@ Film 2050 film. Very high electrical breakdown strengths have been measured on this commercially available silicone elastomer film. Electrical breakdown strength values of $177 \mathrm{~V} / \mu \mathrm{m}$ for $45 \mu \mathrm{m}$ thick film with a Weibull $\beta$-parameter of 37 , and $337 \mathrm{~V} / \mu \mathrm{m}$ for $25 \mu \mathrm{m}$ thick film with an unprecedented Weibull $\beta$ parameter of 40, have been measured[59], with both showing a very narrow distribution of breakdown around very high electrical breakdown strength (as indicated by the large $\beta$ parameter value). Common $\beta$-parameter values for silicone elastomers produced by knife casting are usually around 5-25, and as a rule of thumb, values of the order 5-10 usually indicate inhomogeneous elastomers, whereas values of 20-25 - to date-have been treated as state of art and representing fully homogeneous films. For small-volume transducers, electrical breakdown strength is the most important for good product reliability, but for largevolume transducers, the $\beta$-parameter becomes the most important factor. This is due to rather unlikely events suddenly becoming likely to take place in film volumes approaching infinite values, compared to the measurement volume of the electrical breakdown strength. Therefore, a very narrow distribution is crucial for the reliability of large-volume transducers, and so electrical breakdown plays a less significant role.

\subsection{Discussion on possible pitfalls when formulating composite elastomers}


The addition of high-permittivity filler to commercial elastomer systems is one of the most investigated solutions to the challenge of acquiring improved energy densities, due to its apparent simplicity. Commercial systems, however, are often relatively high in silica content (of the order $30-40 \%$ by volume), and the option to increase overall filler content comes at the price of an inherent increase in the elastic modulus, due to increased particle-particle and particle-network interactions - and thus usually status quo in improving energy density. The same holds for adding fillers to initially non-filled silicone elastomers, but here the starting point for the elastic modulus is significantly lower, and therefore improved actuation may be obtained when comparing to the rather stiff commercial elastomers. However, most often, these self-formulated silicone elastomers are not optimal for compositing, since various compatibilizers are not present in self-formulated silicones. Thus, the real improvement is again limited, and at the same time, the reliable processing of composite elastomers becomes a tremendous challenge, owing to the very high viscosities involved. Due to the irreversible curing of the elastomer premixes, heating cannot be utilized to decrease viscosity, as we see done for thermoplastics. The use of significant amounts of solvents, to some extent, can eliminate viscosity issues, but it introduces many undesirable issues when removing the residual solvent again[47,60].

For the optimal elastomer system in terms of actuation, undesirable reinforcement has to be weighed towards permittivity increase. This is the main focus in Yu and Skov[56], where it was established that the most feasible way forward was to base composites on commercial elastomers for which the amount of silica was reduced. In other words, let the permittivity-enhancing filler partly take over the role of the reinforcing silica. Wacker Chemie AG provided these formulations based on the XLR 630, namely two formulations with $25 \%$ and $50 \%$ less silica filler, respectively. Instead of silica, the "reduced elastomers" were filled with high-permittivity titanium dioxides, in order to obtain the desired improved 
dielectric permittivity. Thereby, the most favorable properties of the commercial elastomers were maintained while improving both dielectric permittivity and electrical breakdown strength.

Despite initial positive results, it turns out that the XLR formulation, even with no silica content reduction, was not a good basis for dielectric elastomers, since it had a very minor rupture at around the $200 \%$ extension. This minor discontinuity in the stress-strain curve was maintained during the change of filler composition and also strengthened in the sense that the minor rupture turned into a significantly larger one. Initially, this was believed to be an issue with the Instron instrument used to measure stress-strain behavior, but the same minor break (an almost vertical drop of approximate $0.5 \%$ in Young's modulus) in the stressstrain curve was also observed in another instrument at approximately the same strain. This drop was ascribed to local weakness in the elastomer, which, however, did not result from inhomogeneous mixing, since it was reproducible. It must rather have been an inelastic component in the commercial elastomer formulation not being able to maintain the stresses generated at approximately $200 \%$ strain. This led to further investigations into tearing properties. It was concluded that this small rupture was problematic, as it could potentially lead to the tearing of thin film elastomer, since the testing geometry for tearing involved significantly thicker samples than when testing for actuation. Nevertheless, the approach of reducing silica content was proven to be feasible, since commercial elastomers in general are filled with much more silica than required, due to the low price of the filler compared to the PDMS matrix. Simply the wrong silicone elastomer was chosen, and this example gives a clear indication of the necessity and importance of fully characterizing and carefully examining the starting elastomer when formulating. The addition of additional filler will not repair any defects inherent in pristine silicone elastomer but rather exacerbate it. 
Further complications arise in processing composite elastomers. Several issues are often ignored in the scientific literature when formulating with high loadings of fillers, i.e. in highly viscous environments, as also discussed in Lotz et al.[61] The first issue addresses if the viscosity of the elastomer premixes decreases as a result of increased loading, which provides a clear indication of poor network structure around the filler (incompatible systems), such that stresses throughout the cured composite will become uneven. In the worst case, visible air voids also arise on large stretches, and such voids will lead to premature electrical breakdown, since air breaks down electrically at around $3 \mathrm{~V} / \mu \mathrm{m}[62]$. Alternatively — and less disruptive immediately - these voids on longer time-scales will facilitate the growth of electrical trees[63] and thus lead to detrimental electrical ageing.

Secondly, homogeneous mixing is not automatically attained, and thus reproducibility is limited, not only locally in the elastomer, but also from batch to batch, and the composite is more likely to suffer from premature failure due to this inhomogeneity. Achieving homogeneous mixing is complicated further by the heat of mixing being rather significant for these highly viscous systems, and thus partial curing takes place during the mixing. In the worst case, macroscopic lumps are obtained. This is illustrated in Figure 7, where SEM pictures are shown of the resulting elastomers from hand-mixing and speed-mixing, respectively, of 2 phr expanded graphite into Elastosil RT625[42].
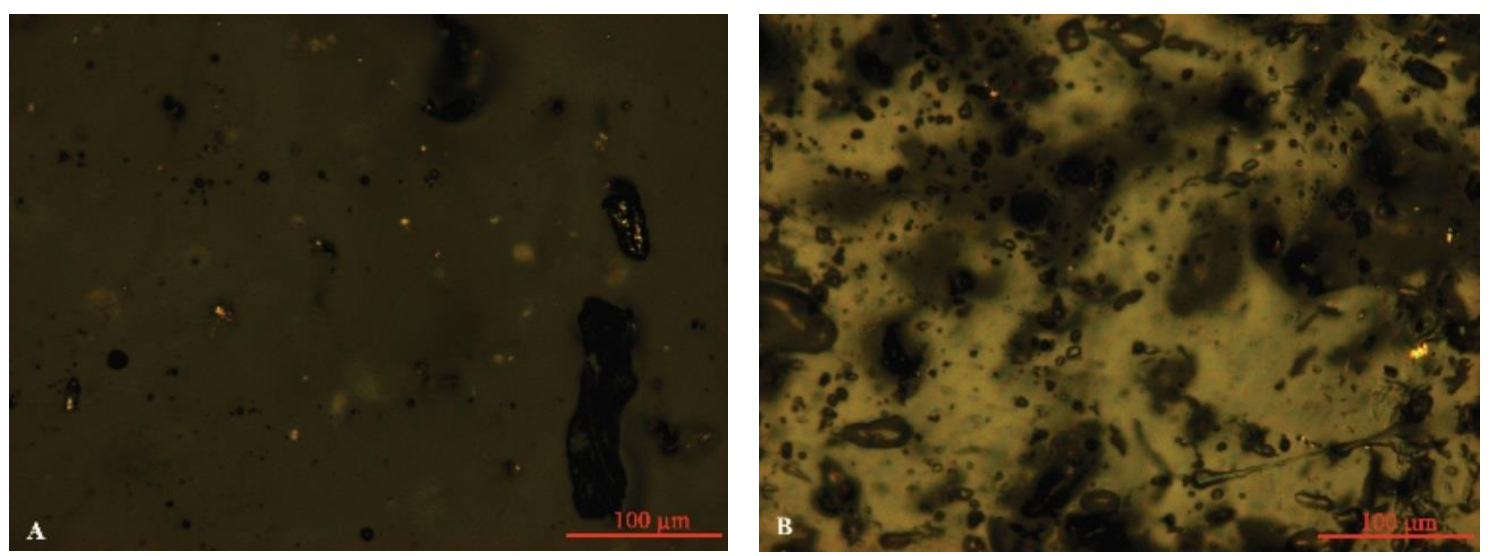
Figure 7: SEM pictures of two identical 2 phr samples (approximately $1 \%$ on volume basis) of expanded graphite (EG) in RT625 prepared by (A) hand-mixing for 10 minutes and (B) speed-mixing two times for 2 minutes. The EG has been dispersed homogeneously in solvent by ultra-sonication before being mixed in for both mixing procedures, but system (A) clearly aggregates. The pictures illustrate the need for proper mixing, in order to achieve composite homogeneity.

Both the Payne and the Mullin effects become significant with increased filler loading, and thus mechanical properties become much more non-linear and strongly timeand stretch-dependent. This puts further requirements on the controlling system of the transducer and is thus very unfavorable for most applications. The permanent set may also become rather significant[45], and again, going back to the discussion on the network structure, a large permanent set is a clear indication of too high a concentration of particulate fillers in the system (the sum of permittivity-enhancing filler and silica) or an inhomogeneous distribution of fillers. The implications of inhomogeneity for commercial elastomers are minor, due to the inherent stability of the formulation of mainly silica and silicone. Of minor constituents by mass but not necessarily by effect are various stabilizers and additives. For compositing with less compatible constituents, inhomogeneities are more likely to occur due to thermodynamically unstable mixtures. Due to large differences in the Young's modulus and ultimate strain of matrices and fillers, inhomogeneity will influence strongly the final electro-mechanical properties.

Summing up, the composite methodology requires an extensive focus on the mixing process, which often includes advanced mixing schemes in order to achieve homogeneous, reproducible, and reliable elastomers. Ensuring and controlling homogeneity is not a trivial undertaking, since optical microscopy and SEM are very complicated to use for highly loaded 
composites, because transparency is lost in the case of different refractive indices, whereas silica particles are almost impossible to detect in a silicone matrix, due to similarities in contrast in SEM/TEM.

\subsection{Composite systems based on soft fillers}

Since silicone polymers are inherently soft and immiscible with most other substances-even on short time-scales - there are, to the best of our knowledge, no examples of silicone elastomers with soft fillers dispersed into silicone elastomers in discrete compartments, except in the case of Mazurek et al.[64-66] In other words, it means that there is phase separation on the microscale or even the nanoscale. The case of blends, i.e. a homogeneous melt of soft fillers dissolved in the elastomer, is discussed later.

In Mazurek et al.[64], work is presented on how to disperse glycerol in silicone premixes in stable emulsions, which are then crosslinked into homogeneous closed-cell structures with glycerol dispersed as discrete droplets. The stability of the emulsion is very counterintuitive, but it remains stable both in simulated injection moulding equipment and can subsequently be heated to $80^{\circ} \mathrm{C}$ in order for crosslinking to take place. The behavior of this composite type lies in between the traditional composites and blends discussed in the subsequent section. This high-permittivity substance (such as glycerol) is enclosed in discrete compartments and ideally should not interfere with the crosslinking reaction.

Many ionic liquids (especially those containing nitrogen) inhibit the Pt-catalysed crosslinking reaction and are therefore not suitable candidates without changing the elastomer to, for example, a condensation-type silicone elastomer that is not prone to inhibition to the same extent. The properties of this system are discussed in reference[64], and the morphology of a moderately filled ( $34 \%$ by volume) glycerol-silicone elastomer is illustrated in Figure 8. This system can be considered as encompassing foams with a closed cell structure, where the cells are liquid-filled, which is in contrast to earlier work by Hunt et 
al.[67], in which they prepared open cell foams filled with silicone oil, to attain self-healing properties. Such a structure would give rise to the mobility of high-permittivity liquid.

Summarizing these results, this soft composite type, for which a scaling analysis is performed below, has shown very promising results.

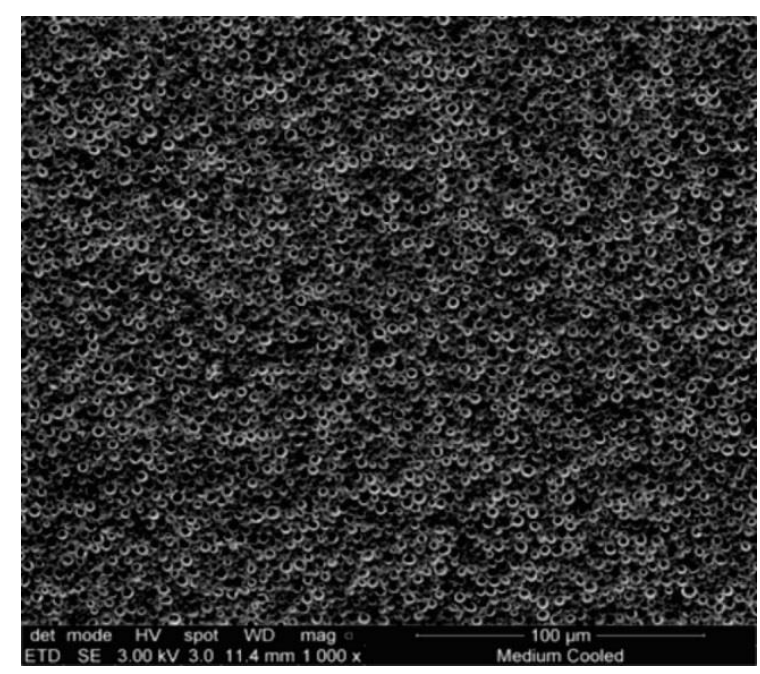

Figure 8: SEM picture of a crosslinked glycerol-silicone elastomer composite for $60 \mathrm{phr}$ glycerol (approximately $34 \%$ by volume) in Sylgaard 184 elastomer. The glycerol is dispersed evenly as discrete droplets throughout the elastomer matrix.

The Young's modulus of this type of composite arises solely from the elastomer matrix, which is diluted with the volume fraction of the filler, since the dispersed liquid does not contribute to elasticity:

$$
Y=Y_{0}\left(1-\Phi_{\text {filler }}\right)
$$

Dielectric permittivity is assumed to follow the same trend as for the composites based on hard fillers (Equation 8). Thus improved actuation at a given voltage can be written as:

$$
\frac{\epsilon_{r}}{Y} / \frac{\epsilon_{r, 0}}{Y_{0}}=\frac{1+k_{\epsilon} \Phi_{\text {filler }}}{1-\Phi_{\text {filler }}}
$$


The breakdown strength of the composite is assumed crudely, to follow the trend derived in Equation 11, i.e. electrical breakdown strength decreases in line with increased loading, due to the composite softening. Thus, the figure of merit for the generator can then be written as:

$$
\frac{\epsilon_{r} E_{B D}^{2}}{\epsilon_{r, 0} E_{B D, 0}^{2}}=\left(1+k_{\epsilon} \Phi_{\text {filler }}\right)\left(1-k_{B D}^{\star} \Phi_{\text {filler }}\right)^{2}
$$

The two figures of merit are shown in Figure 9 for glycerol-loaded soft composites. For glycerol, with $k_{\epsilon} \simeq 60 / 3 \simeq 20$ and $\Phi_{\text {filler }} \simeq[0,0.5]$, it is obvious that these elastomers hold great promise as both actuators and generators, since both figures of merit (Equations 15 and 16) are significantly larger than 1 . This also holds for other commonly used liquids with high dielectric permittivity, such as water, propylene carbonate, and ionic liquids. The addition cured silicone elastomer and glycerol system is by far the best soft composite system investigated to date, since the glycerol and silicone phases separate very easily and are easy to manipulate and control. Furthermore, the vapor pressure of glycerol at room temperature and in curing conditions vanishes, and the glycerol does not inhibit the curing reaction when addition or condensation curing elastomers are used. However, the method may be investigated further in the future and reveal better potential by using, for example, suitable ionic liquids as high-permittivity soft fillers.

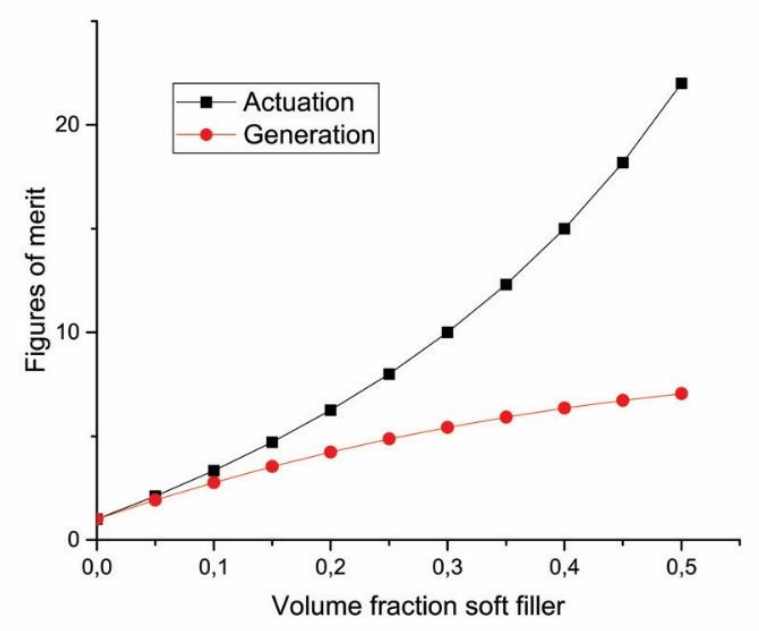


Figure 9: The two figures of merit for the glycerol-PDMS soft composite system with the experimental determined values of $k_{B D}^{\star}=0.4$ and $k_{\epsilon}=20$. For higher-permittivity liquids, such as ionic liquids, an even greater improvement in figures of merit could be achieved.

The major limitation of this approach is that the incorporated liquid needs to be highly non-volatile in the curing conditions set up for the silicone elastomers, and vapor pressure should be insignificant at room temperature. Water $\left(\epsilon_{r}=80\right)$, for example, evaporates fully from a silicone elastomer matrix within a few days at room temperature. Other liquids that were incorporated successfully were propylene carbonate $\left(\epsilon_{r}=65\right)$ and various ionic liquids $\left(\epsilon_{r}=10-80\right)$, but film quality was poor, due to the inhibition of the curing reaction compared to the excellent films formed with glycerol as a soft filler. Potentially, identifying suitable combinations of ionic liquids and elastomers may hold great promise.

\section{Silicone/polymer blends}

From the scaling analysis performed earlier, it is obvious that with current formulation techniques, dielectric permittivity cannot be increased by means of metal oxide composites without a simultaneous increase in the Young's modulus, if mechanical integrity is to be maintained. A methodology comparable in simplicity with compositing involves blending in high-permittivity polymers of various compositions, structures, and molecular weights [68]. In contrast to the soft, dispersed fillers, here miscibility is sought such that the blends are completely homogeneous. The polymers that need to be blended in should be non-reactive, in order not to chemically influence the network structure, and thus they are denoted as oils in the following. Such high-permittivity oils concurrently soften the elastomer and increase dielectric permittivity, and thereby electromechanical responses increase significantly.

\subsection{Empirical descriptions of blend system figures of merit}


The elastic modulus will scale with the volume fraction of the network $\left(\Phi_{\text {network }}\right)$, as shown experimentally and discussed phenomenologically in Larsen et al.[69]:

$$
Y=Y_{0} \Phi_{\text {network }}^{n}=Y_{0}\left(1-\Phi_{\text {oil }}\right)^{n}
$$

where $Y_{0}$ is the Young's modulus of the elastomer with no oil, and $n$ is a scaling parameter between 1 and 2.4, as derived in reference[69]. Nonlinearity is due to the simultaneous dilution of crosslinks (linear relation between $\mathrm{Y}$ and dilution) and a reduction in trapped and locked entanglements (binary interactions, thus scaling the dilution to the second power). For non-entangled polymers $n=1$, and for highly entangled networks $n \simeq 2.4$ as a rule of thumb. Most silicone elastomers, however, are well entangled, since silicone elastomers from polymers close to or below the entanglement molecular weight become brittle.

Permittivity increases approximately linearly when filling with high-permittivity oil, and the resulting figure of merit follows on from that of the previous section:

$$
\epsilon_{r}=\epsilon_{r, 0}\left(1-\left(1-k_{\epsilon}\right) \Phi_{o i l}\right) \simeq \epsilon_{r, 0}\left(1+k_{\epsilon} \Phi_{o i l}\right)
$$

where $k_{\epsilon}=\epsilon_{r, o i l} / \epsilon_{r, 0}>1$ is a dimensionless proportionality factor.

Thus, improved energy density as a function of the volume fraction of oil can be written as:

$$
\frac{\epsilon_{r}}{Y} / \frac{\epsilon_{r, 0}}{Y_{0}}=\frac{1+k_{\epsilon} \Phi_{o i l}}{\left(1-\Phi_{o i l}\right)^{n}}
$$

The equation above states that energy density can actually be increased perpetually by letting the volume fraction of oil approach 1 (due to the simplification of assuming the permittivity of the oil as being significantly larger than that of the silicone elastomer). Of course, this does compromise network integrity, and the upper limit for $\Phi_{\text {oil }}$ will be discussed in the following. The relation is plotted in Figure $\mathbf{1 0}$ for various realistic combinations of the parameter $k_{\epsilon}$ for a well-entangled network $(n=2.4)$. Examples of relatively compatible oils could be chloropropyl functionalized silicone oils $\left(k_{\epsilon} \approx 2[70]\right)$, flouro-functional and 
phenyl-functional silicone oils $\left(k_{\epsilon} \approx 5[71,72]\right)$, and various benzo-functional oils $\left(k_{\epsilon} \approx\right.$ 10[73]).

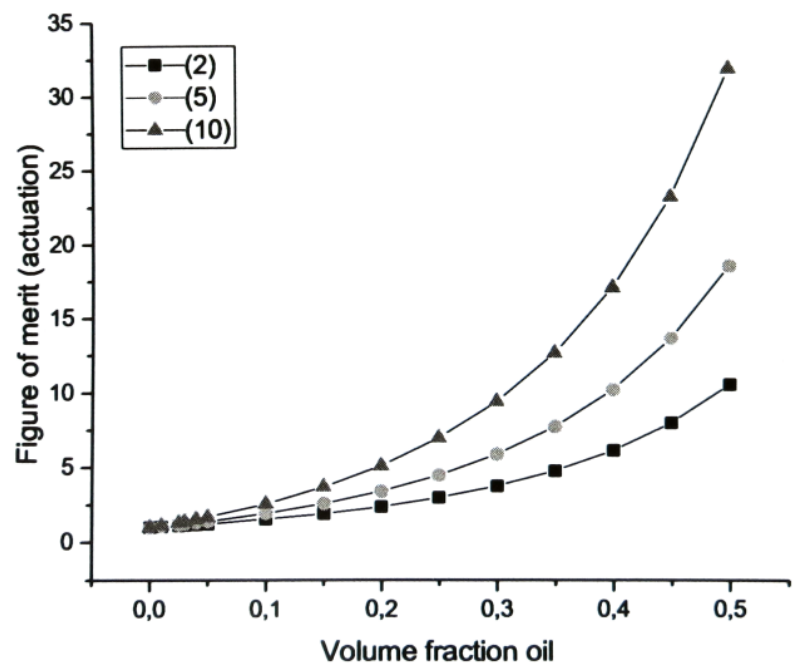

Figure 10: Figure of merit in actuator mode for blends with varying values of $k_{\epsilon}$ stated in the legend. The given values can correspond to e.g. chloropropyl functionalized silicone oils $\left(k_{\epsilon} \approx 2\right)$, phenyl-functional silicone oils $\left(k_{\epsilon} \approx 5\right)$, and benzo-functional oils $\left(k_{\epsilon} \approx 10\right)$.

With the very positive prospect for silicone/polymer blends being utilized as actuators, the picture changes when discussing generators. Electrical breakdown strength was shown to decrease significantly in line with oil content[74], and thus the obtained improvement from adding oil may be rather limited when not comparing in a constant electrical field. The following empirical relation was found to hold for chloropropylfunctional silicone oil in a high electrical breakdown strength commercial elastomer (LR 3040/50 from Wacker Chemie):

$$
E_{B D} / E_{B D, 0} \simeq 1-\frac{1}{200} p h r
$$


where phr is parts (oil) per hundred parts elastomer. Despite convenience when formulating, the unit does not provide much direct information on the more physically relevant fractions, such as volume and weight.

Equation 20 states that electrical breakdown strength approaches 0 when $p h r \rightarrow 200$, i.e. $\Phi_{\text {oil }} \rightarrow 2 / 3$. This also agrees well with the upper limit for loadings of fully compatible oils where films are no longer freestanding at this approximate loading. Nevertheless, this equation is fully empirical and may hold only for the particular LR 3040/50 system, a very tough elastomer, with chloropropyl-functional silicone oil. For this system, a small increase in electrical breakdown strength was also noted when utilizing a small amount of oil. This was ascribed to the reduction in viscosity that facilitated easier coating, and thus better films were obtained than with the corresponding oil-free films.

Translation of the engineering unit $p h r=100 m_{\text {oil }} / m_{0}$ into volume fractions is done by assuming that the initial partial volumes are additive $\left(\Delta \mathrm{V}_{m i x}=0\right)$ :

$$
\Phi_{\text {oil }}=\frac{m_{\text {oil }} / \rho_{\text {oil }}}{m_{\text {oil }} / \rho_{\text {oil }}+m_{0} / \rho_{0}}=\frac{m_{\text {oil }} / m_{0}}{m_{\text {oil }} / m_{0}+\rho_{\text {oil }} / \rho_{0}} \simeq \frac{p h r}{p h r+100}
$$

where $m$ and $\rho$ are the masses and densities, respectively, of the pure elastomer (subscript 0 ) and the oil (subscript oil). It is assumed furthermore that the densities of oil and elastomer are approximately equal.

Solving for $p h r$, we get:

$$
p h r=\frac{\Phi_{\text {oil }}}{1-\Phi_{\text {oil }}} \cdot 100
$$

Thereby, the following expression for electrical breakdown strength in terms of volume fraction is obtained:

$$
E_{B D} / E_{B D, 0}=1-\frac{\Phi_{o i l}}{2\left(1-\Phi_{o i l}\right)}=\frac{1-3 / 2 \Phi_{o i l}}{1-\Phi_{o i l}}
$$

From Equations 18 and 23, the expression for improved generator performance follows as: 


$$
\frac{\epsilon_{r} E_{B D}^{2}}{\epsilon_{r, 0} E_{B D, 0}^{2}}=\left(1+k_{\epsilon} \Phi_{o i l}\right)\left(\frac{1-3 / 2 \Phi_{o i l}}{1-\Phi_{o i l}}\right)^{2}
$$

This function is plotted in Figure 11. It is obvious that in order for the method to provide a significant improvement, the relative dielectric permittivity of the oil has to be rather high (high $k_{\epsilon}$ ). However, when this is achieved, the oil departs from its silicone-like properties, in which case miscibility is expected to become an issue. Another interesting finding is that the volume fractions have to lie in the range of 0.3 to 0.4 for the best results, which is also the range where the best film-forming properties are obtained. This was, however, ignored in the breakdown strength fit, so the result further supports the ideality of the given elastomer system with $30 \%$ to $40 \%$ silicone oil. To our surprise, elastomers based on 30\% with chloropropyl-functional PDMS oil were very stable over time when static prestretching[75].

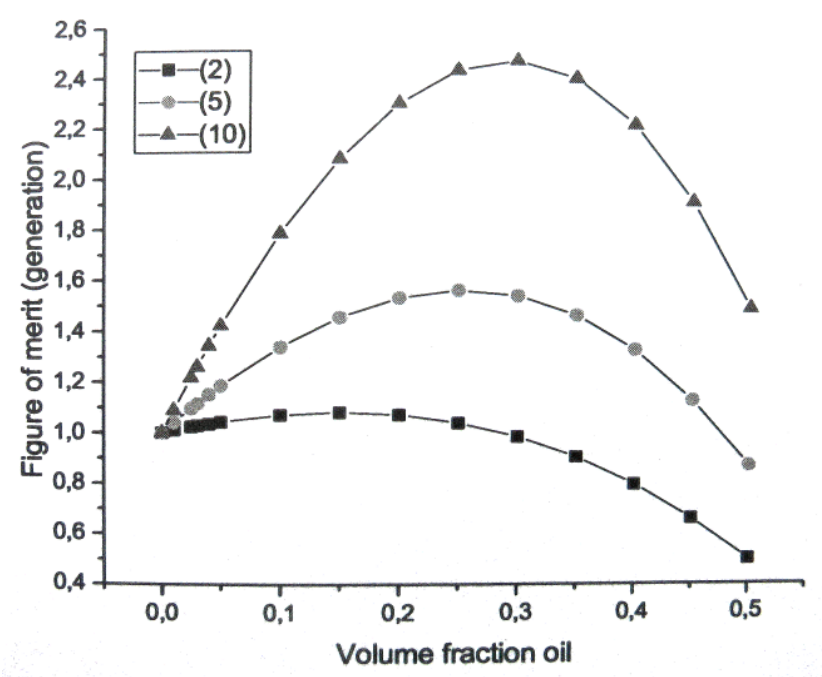

Figure 11: Figure of merit in generator mode for blends with varying values of $k_{\epsilon}$ stated in the legend. The given values can correspond to e.g. chloropropyl functionalized silicone oils $\left(k_{\epsilon} \approx 2\right)$, phenyl-functional silicone oils $\left(k_{\epsilon} \approx 5\right)$, and benzo-functional oils $\left(k_{\epsilon} \approx 10\right)$.

\subsection{Discussion on possible pitfalls when formulating elastomer blend formulations}


Following our guideline (based on our experience of silicone elastomer formulation) of approximately one-third of the silicone elastomer replaced with oil as the maximum allowable amount to increase electro-mechanical properties, ultimate properties are changed as well, whereas elastomer stability usually remains. Tensile strength decreases by approximately one-third (assuming that the tensile strength scales with crosslinking density), but since the original elastomer for this approach is - and needs to be - very strong, this does not pose a problem for most practical applications. It does, however, give an indication of which elastomer systems are suitable for this method. Very tough and durable elastomers are to be used for the blending method for two obvious reasons. First, their ultimate mechanical properties will be affected by the oil, and second, the decrease in electrical breakdown strength with oil loading may be even more significant for a soft starting material. As an example, the rather soft Elastosil RT625 cannot accommodate $60 \%$ silicone oil without becoming gel-like; it remains elastic upon actuation but is very weak and suffers from significant hysteresis. On the other hand, Elastosil RT625 (Y=1 MPa determined at 5\% strain[76]) can easily accommodate $30 \%$ of silicone oil and still maintain its mechanical integrity[77]. Elastosil RT625 with 15\% silicone oil has been studied extensively in various products, since it was the elastomer system utilized in the film production of the Danish company Danfoss PolyPower, which was one of the first enterprises to produce dielectric elastomers including electrodes on a commercial scale. No oil leakage was observed in an extensive series of tests both on the pristine films and in products.

Many different classes of permittivity-enhancing oils have been utilized, but almost all elastomers will eventually fail following the migration of the oil out of the elastomer, due to the incompatibility of all polymers with PDMS. Of course, such failures may not be seen within the preparation and testing time, but they will appear on the timescale of weeks, months, or even years. This behavior seems rational when considering how difficult it is to 
obtain a homogeneous mixture of two rather immiscible polymers. To facilitate dispersion, often as a minimum, surfactants are utilized to increase compatibility. However, most of the silicone compatible surfactants tested in our laboratory were found to have a negative impact on electrical breakdown strength, most likely due to migration in the electrical field[78-80].

If an elastomer composition with significant amounts of oil is chosen for dielectric elastomer transducers, it is very important to consider the long-term stability of not only dielectric elastomer, but also the interface between dielectric elastomer and electrodes. If the oil migrates, it will first move to the surface of the dielectric elastomer and thereby to the interface between the electrode and the elastomer. An oily layer here may result in delamination of the electrodes from the elastomer. Alternatively, the oil may migrate further out in the transducer and cause other problems, due to the ability of silicone to spread effectively over most surfaces[81].

To overcome the immiscibility issue, a natural path forward involves using chemically modified PDMS oils, in order to help the PDMS allow for miscibility and functional moiety, thus ensuring increased dielectric permittivity.

\section{Chemically modified silicone elastomers}

The chemical modification of silicone elastomers is a much more demanding and tedious approach than the two previous methods. The general idea is to replace some of the methyl groups of the simplest silicone, namely polydimethylsiloxane (PDMS), with high-permittivity moieties, as illustrated in Figure 12, and then functionalize the end-groups to allow for crosslinking[82-84]. Some approaches also use the end-groups for chain extension, but the final product remains a telechelic functionalized silicone polymer. 


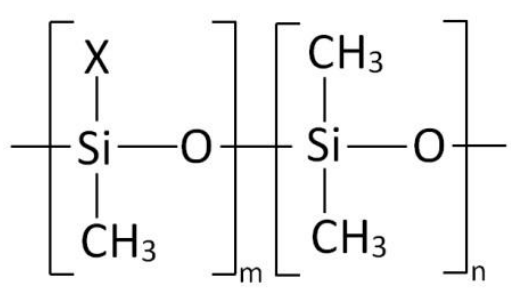

Figure 12: Illustration of a common chemically modified silicone polymer structure, where $\mathrm{X}$ denotes high permittivity moiety. The ratio $\mathrm{m} /(\mathrm{m}+\mathrm{n})$ gives the degree of substitution.

The chemical modification methodology relies on covalent grafting of non-silicone entities onto the silicone backbone, in which case the migration of immiscible components will be hindered. Alternatively, the crosslinkers can be modified, but this approach dramatically decreases the achievable concentrations of high-permittivity filler[85,86]. Alternatively, functionalized chain extenders can be utilized, but this approach complicates formulation[87]. Grafting often requires complex and novel chemistries, since the chemistry of silicones is rather unexplored. Examples of well-known chemistries include the PiersRubinsztajn and Huisgen reactions[88-91].

By employing the covalent grafting method, immiscibility issues are avoided, of course provided that the reaction mixture was homogeneous initially and the curing did not alter this state.

\subsection{Empirical descriptions of chemically modified elastomer figures of merit}

Chemically modified silicones allow for maintaining the Young's modulus, i.e. they help maintain mechanical integrity. This relies on the mere fact that the volume of the grafted molecules is usually rather limited, especially when comparing to the methyl group of the silicone, which is replaced by grafted moiety. Furthermore, the grafting approach should preferably allow for orthogonal, non-interfering reactions such that the subsequent crosslinking reaction is not influenced. As a result, crosslink density does not change 
significantly when comparing to a reference consisting of the same crosslinked polymer chain but without the grafted moieties.

Consequently, for such elastomers, the improvement factor can be written easily as the contribution from the permittivity enhancement, which is assumed to increase linearly in line with loading — as seen for the previously discussed approaches:

$$
\frac{\epsilon_{r}}{Y} / \frac{\epsilon_{r, 0}}{Y_{0}}=1+k_{\epsilon} \Phi_{c h e m}
$$

Moreover, with electrical breakdown strength remaining more or less unaffected, the generator figure of merit becomes:

$$
\frac{\epsilon_{r} E_{B D}^{2}}{\epsilon_{r, 0} E_{B D, 0}^{2}}=1+k_{\epsilon} \Phi_{c h e m}
$$

In this methodology, the amounts of chemical substituents are limited if the silicone nature of the elastomer needs to be maintained. In other words, the method can only lead to incremental increases in the figures of merit, if not based on a very high permittivity substituent. However, there are many reasons for why, if one is looking to investigate this area further. An obvious fact is that when the covalent anchoring of molecules into the elastomer backbone occurs, the elastomer will not be prone to the long-term migration of any species within the network structure. Furthermore, another interesting property of theseusually aromatic - substances, namely the ability to increase electrical breakdown strength, is so-called "voltage stabilization", first observed in silicone elastomers for the chloropropyl functionalized elastomers[92]. The chloropropyl moiety deserves an individual discussion, since the dual behavior of this moiety is rather interesting and holds great promise.

\subsection{Further functions of chemically grafted moieties to the silicone backbone}

Until now, the effect of incorporating various voltage stabilizers onto the silicone backbone on the electrical breakdown strength of dielectric elastomers has not been investigated. Voltage stabilizers are well known in the high-voltage cable industry, where most interest has 
centered on stabilizing (i.e. increasing the electrical breakdown strength of) polyethylene (PE), the most commonly used material for high-voltage cables[93-95]. The principle relies on the addition of minute amounts of moieties that absorb the electrons with energies otherwise capable of participating in the various breakdown mechanisms involved in shortcircuiting the polymer. For PE, dramatic increases of up to about $50 \%$ in electrical breakdown strength have been achieved. A study in our laboratories shows that subpercentage additions of various aromatic substances can increase electrical breakdown strength significantly, but this is not yet comparable with improvements achieved with $\mathrm{PE}[96,97]$. Increases in electrical breakdown strength of up to $10 \%$ have been achieved by adding $0.1 \mathrm{wt} \%$ of an aromatic moiety covalently grafted to the silicone backbone. This is of course not a paradigm-changing result, but nevertheless it does move in a direction towards a feasible and significant improvement to dielectric elastomers, which are limited by their electrical breakdown strength. Another example of voltage-stabilized silicone elastomers is the commercially available Elastosil@ Film 2050 from Wacker, as discussed earlier. This film has surprisingly high electrical breakdown strength, and even a small $10 \%$ increase in this regard would lead to a 1.21 improvement in the figure of merit for energy generation. In addition, it remains to investigate the influence of voltage stabilizers on electro-mechanical stability over time; here, the effect may be even more significant.

\subsection{Particular properties of chloropropyl groups covalently grafted to the silicone backbone}

A rather well-investigated system is silicone elastomers with chloropropyl groups covalently grafted to the PDMS backbone, as shown in articles[92,98,99]. The chloropropyl group was initially introduced solely to allow for further functionalization. The dielectric permittivity of the chloropropyl group was considered uninteresting, but measurements performed on this reference system revealed very promising results. First, a small increase in dielectric permittivity was achieved, but second, the Young's modulus decreased rather significantly 
while the elastomer maintained its mechanical integrity. Further investigations revealed that crosslinking density remained more or less unaffected, i.e. the sole reduction in crosslinking density was due to the introduction of the bulky chloropropyl groups (additional swelling experiments confirmed this point). Finally, the electrical breakdown strength behavior followed that of the oil-filled systems with an intermediate optimum in loading of the substituent/oil. These observations led us to the conclusion that the bulky chloropropyl groups were self-lubricating, i.e. intermolecular chain frictional forces are reduced, possibly due to increased free volume. This observation paves the way forward for better elastomers with respect to actuation performance. The lubrication process is similar to that of the entanglement reduction method, which will be discussed later.

Further studies on this system also revealed that chloropropyl groups, whether covalently grafted or present as oil, influence dielectric loss, which is substantially reduced

with the content of chloropropyl groups[98]. The mechanism for this is still not understood, but nevertheless, it works for a wide range of composite systems. Yet another feature is that for certain chloropropyl group concentrations, the electrical breakdown mechanism changes and the subsequent tearing of films can be reduced[76]. Microscopic electrical breakdown followed by macroscopic tearing is one of the major challenges to producing large-quantity dielectric elastomer transducer products, since a local electrical breakdown taking place under large strains will immediately lead to a significant destruction of the dielectric elastomer film, if precautions are not taken.

\subsection{Discussion on the possible pitfalls of formulating with chemically modified elastomers}

The formulation of elastomers based on covalently chemically modified elastomers is straightforward and facile. Processing does not differ from the traditional formulation process, but one precaution has to be taken with respect to ensuring homogeneity, such that 
there is no microscopic phase separation before curing. This can, however, be ensured easily by speed-mixing or other strong shear techniques[100,101] and then using suitable solvents.

Of course, the difficulty in this methodology lies in the preparation of the chemically modified moieties to be grafted onto the silicone backbone. Since the tendency is towards more and more specialized silicone entities being commercialized, such as by Gelest[102], it also becomes easier to prepare the elastomers. The chemical complication lies in introducing a moiety while maintaining the miscibility of the reactants and the crosslinking functionalities, or alternatively introducing crosslinking facilities without affecting the moiety. In addition, many moieties have an inhibiting effect on the platinum-catalyzed reactions[103].

Overall, chemical modification reduces the need for the extensive testing of phase separation during storage and under the influence of an electrical field and oil migration, as mentioned for some of the other permittivity-enhancing approaches. The major trade-off in this respect lies in either increased direct costs or increased indirect costs as a result of tedious synthesis work.

\section{Quantitative comparison of the four permittivity-enhancing methods}

From the scaling relations of the various permittivity-enhancing approaches, elastomers from two methods stand out as actuator material candidates, namely composites with soft fillers and blends.

Correspondingly, from these results it is obvious that there is no ideal, universal dielectric elastomer candidate; rather, operational conditions dictate the ideal elastomer. In the current discussion, the influence of strain, strain rate, and frequency on the various parameters has also been ignored. If taking these into account, it is obvious that multiple elastomers are required to cover optimally a broad range of applications. For example, loudspeakers operate in the upper $\mathrm{kHz}$ range, with little displacement, and are preferably 
lossy at the operating frequency[104,105]. Another example is wave energy harvesting units, which rely on ocean waves with frequencies below $0.1 \mathrm{~Hz}$ and very large strains[106,107]. Losses will lead to element heating and may result in thermal breakdown[108,109]. Of course, both systems can be geared and modified to comply with more intermediate frequencies and strains, but modification takes out the simplicity of the dielectric elastomer's operational principle and furthermore decreases energy efficiency.

Besides the measures in the figures of merit, there are many other aspects to take into account when deciding on a suitable method for formulating a given dielectric elastomer. In Table 1, various aspects of the three methods are summarized, in order to get an indication of some of the pros and cons of the various methods while omitting the figures of merit discussed in detail earlier.

As a guideline towards which chemistries to utilize in the different approaches a summary has been made in Table 2. In general, the condensation cure chemistry is more robust but control over network structure is lost since the reaction schemes involved in condensation lead to a plethora of structures and issues with reproduction if humidity levels are not controlled very strictly.

Finally, dielectric and mechanical losses and current leakage have been ignored in the above figures of merit. Losses are not directly included in the expressions but rather indirectly by limiting filler loadings for which losses become significant (by using rules of thumb). For more details on this relatively unexplored topic of losses in dielectric elastomers, refer to[56].

Table 1: Comparison on how the various approaches affect the stages of film production, and how the films behave on longer timescales.

\begin{tabular}{|c|c|c|c|}
\hline Property/Method & Composite & Blend & Functionalized \\
\hline
\end{tabular}




\begin{tabular}{|c|c|c|c|}
\hline Polymer synthesis & No & No & Yes \\
\hline Mixing/processing & Difficult & Easy & Easy \\
\hline Curing & Easy & Easy & Moderate \\
\hline Film handling affects properties & Yes & No & No \\
\hline Ageing upon static stretching & Moderate & Little & None \\
\hline $\begin{array}{c}\text { Changes in homogeneity upon } \\
\text { applying an electrical field }\end{array}$ & None & Moderate & Little/none \\
\hline
\end{tabular}

Table 2: Formulation strategies and their compatibilities with the two main curing chemistries. $\mathrm{x}$ indicates that the curing mechanism is compatible with the given methodology. (x) indicates that approach and curing is most commonly compatible. - indicates inhibition of catalyst or general incompatibility of approach and curing.

\begin{tabular}{|c|c|c|}
\hline Improvement technique & $\begin{array}{l}\text { Addition } \\
\text { curing }\end{array}$ & $\begin{array}{l}\text { Condensation } \\
\text { curing }\end{array}$ \\
\hline $\begin{array}{l}\text { Compositing with } \\
-\quad \text { metal oxides } \\
-\quad \text { glycerol } \\
-\quad \text { ionic liquids } \\
\end{array}$ & $\begin{array}{l}\mathrm{X} \\
\mathrm{x} \\
-\end{array}$ & $\begin{array}{l}\mathrm{x} \\
(\mathrm{x}) \\
\mathrm{X}\end{array}$ \\
\hline $\begin{array}{l}\text { Blends with oils with } \\
-\quad \text { aromatic/N/S moieties } \\
-\quad \text { non-aromatic/N/S moieties } \\
\end{array}$ & $\begin{array}{l}- \\
\mathrm{x}\end{array}$ & $\begin{array}{l}\mathrm{X} \\
\mathrm{X}\end{array}$ \\
\hline $\begin{aligned} \text { Functionalized elastomer with } \\
-\quad \text { aromatic/N/S moieties (low concentration) } \\
-\quad \text { aromatic/N/S moieties (high concentration) } \\
-\quad \text { non-aromatic/N/S moieties } \\
\end{aligned}$ & $\begin{array}{l}(\mathrm{x}) \\
- \\
\mathrm{x}\end{array}$ & $\begin{array}{l}\mathrm{x} \\
\mathrm{x} \\
\mathrm{x}\end{array}$ \\
\hline $\begin{array}{l}\text { Bimodal elastomers } \\
-\quad \text { regular (small effect on Y) } \\
-\quad \text { heterogenous (large effect on Y) } \\
\end{array}$ & $\begin{array}{l}x \\
x\end{array}$ & $\begin{array}{l}\mathrm{X} \\
- \\
\end{array}$ \\
\hline Bottlebrush elastomers & $\mathrm{x}$ & - \\
\hline
\end{tabular}

\section{Systems with a complex network structure}

As opposed to the previously discussed methodologies increasing dielectric permittivity, the methodology for decreasing the Young's modulus is another obvious approach to improving 
achievable actuation at a given voltage from the equation of actuation (Equation 2).

However, when discussed in the context of reliability, most of the methods for reducing the Young's modulus of silicone elastomers fail. The most common method is to use offstoichiometric reactants to create networks with a significant fraction of dangling substructures and sol, as illustrated in Figure 13. This approach, however, cannot fulfil requirements for the reliability of dielectric elastomers, since their elastic modulus - in the case of reliable materials - is still limited to a lower bound of approximately $0.2 \mathrm{MPa}$, due to the entanglement plateau[69,110,111]. Furthermore, non-stoichiometric elastomers have significant viscous properties, and therefore various relaxations on the timescale from seconds to weeks will be forthcoming. As a result, the simple elastic response of an ideal elastomer is destroyed and time dependency has to be accounted for. Moreover, as discussed earlier, viscous relaxation will lead to losses, which in turn may affect product reliability. In order to overcome these issues, radically different thinking is required.
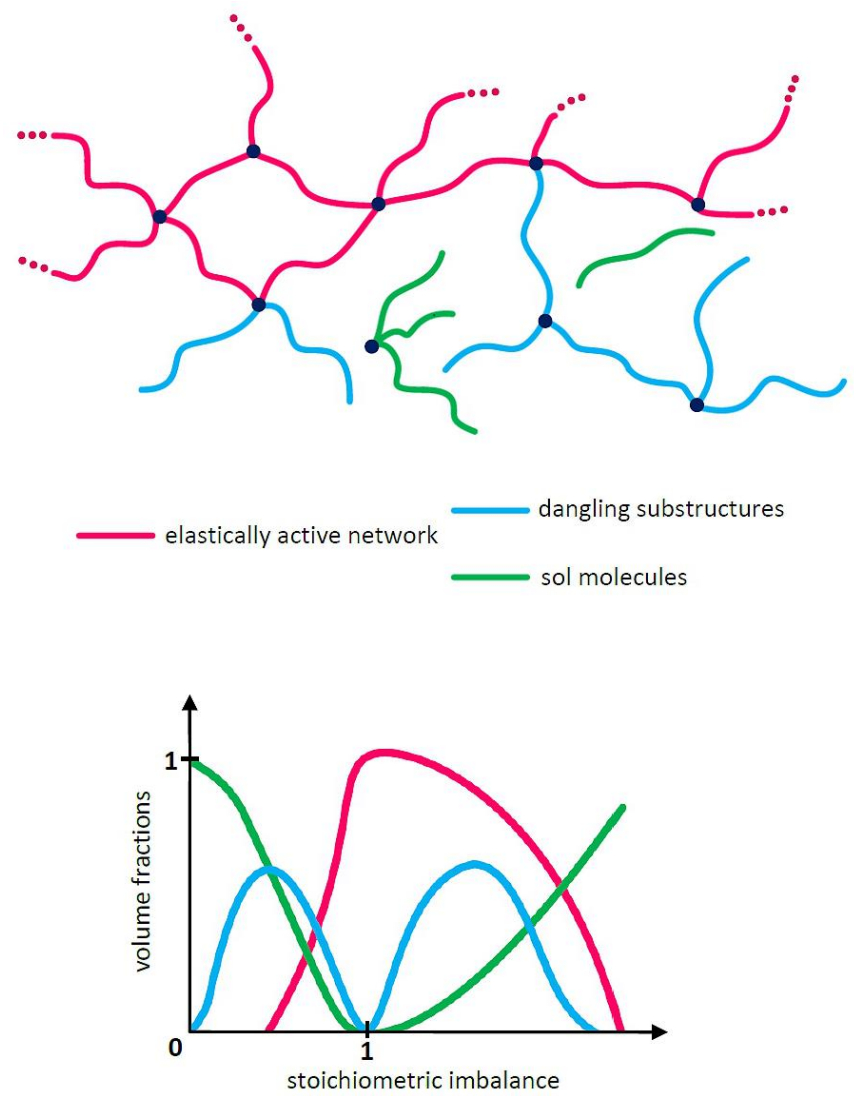
Figure 13: Illustration of various network structures. For ideal networks, all polymer chains are elastically active (pink), i.e. connected at both ends to the infinite network. In real networks, dangling substructures (mainly dangling chains) and so-called "sol polymer chains" exist[112,113]. Dangling substructures are defined as being attached covalently to the infinite network structure at one end only. Sol molecules, on the other hand, are not attached covalently to the infinite network structure.

The idea behind the heterogeneous bimodal network methodology is to decrease the Young's modulus without destroying mechanical integrity — and thus ultimate properties - as discussed in Madsen et al.[114] In other words, soft and very extensible elastomers are synthesized, as shown in Figure 14, and rather than relying on reinforcement by silica particles, reinforcement is introduced by controlled heterogeneity in the network structure, and the resulting elastomers are denoted heterogeneous bimodal elastomers, or just as bimodal elastomers, in the following.

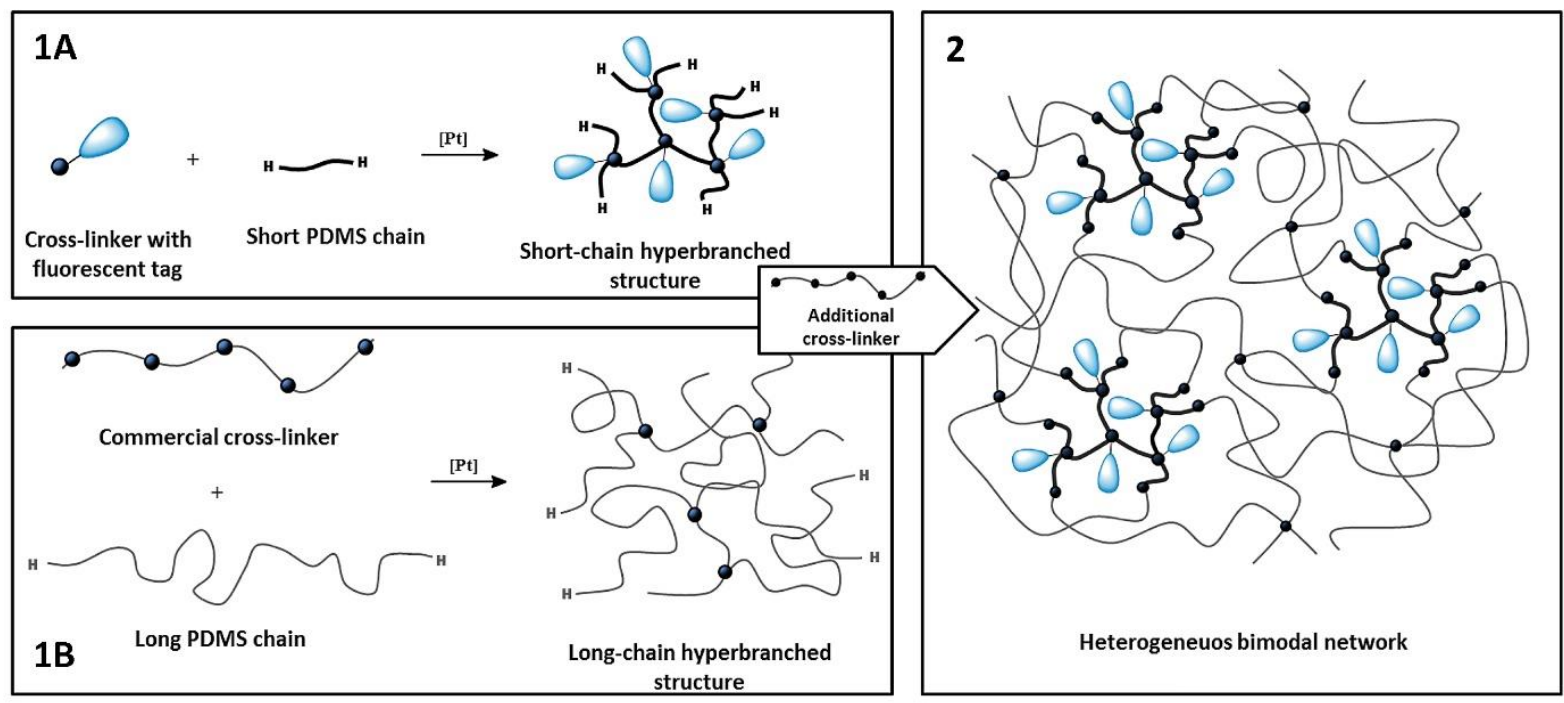

Figure 14: Illustration of the synthetic strategy for achieving heterogeneous bimodal elastomers. The fluorescent tags are used to visualize the domains by fluorescence microscopy. Adapted from reference[114]. 


\subsection{Empirical descriptions of figures of merit for elastomers with a complex network} structure

Improved actuation performance can be written easily, with the sole contribution coming from the change in elasticity described by the constant $k_{Y}^{\star}=Y / Y_{0}$ :

$$
\frac{\epsilon_{r}}{Y} / \frac{\epsilon_{r, 0}}{Y_{0}}=\frac{1}{k_{Y}^{\star}}
$$

Realistic decreases in the Young's modulus are up to around 10-20 times (i.e. $1 / k_{Y}^{\star}=10-20$ ) by means of the methodology for preparing heterogeneous bimodal elastomers. This is definitely a significant improvement, but one should also keep in mind that as a result of this approach, the holding force and tensile force decrease to approximately the same extent as the Young's modulus. At higher decreases, the favorable properties of silicone elastomers, such as high actuation speed and minimal hysteresis, diminish.

An alternative approach to lowering the Young's modulus is the recent methodology initially introduced by Weitz et al.[115,116] and then further elaborated by Sheiko et al.[117], whereby brush-like silicone polymers are prepared and crosslinked. The brush-like structure suppresses the effect of entanglements, since the effective entanglement length increases significantly. These elastomer compositions were tested in our laboratories with respect to their electro-mechanical properties. The formulations possessed very low electrical breakdown strength. The largest decrease in the Young's modulus was $1 / k_{Y}^{\star}=4.5$ with a decreased relative breakdown strength $E_{B D} / E_{B D, 0}=1 / 3$. For actuation purposes, it is obvious that this method - at first glance-works well, but for generator performance, no improvement is achieved $\left(F_{\text {om,gen }}=4.5 \cdot(1 / 3)^{2}=0.5\right)$. The dependency of electrical breakdown strength on the Young's modulus is stronger for this type of elastomer. Though this agrees well with the dynamic material (dangling chains/substructures and sol chains) not 
providing electrical resistance of the same magnitude as the crosslinked polymer chains. For brush-like elastomers, the dangling chain fraction is purposely extremely high, in order to create the effect of increased entanglement length, and the resulting crosslinking density becomes very low, due to the bulky nature of the crosslinked chains. Thus, the elastomer will act dynamically under the influence of a static electrical field and culminate in low resistance to electrical breakdown.

Concerning the evaluation of the performance of the bimodal elastomers as generators, their decreased moduli will also naturally lead to lower electrical breakdown strength. Utilizing the expression from Equation 11, coupling electrical breakdown strength to the Young's modulus gives the following:

$$
E_{B D} / E_{B D, 0}=1+k_{B D}^{\star}\left(\frac{Y}{Y_{0}}-1\right)
$$

Giving a realistic estimation of the constant $k_{B D}^{\star}$ would mean that a ten-fold reduction in the Young's modulus would lead to a reduction of $25 \%$ in electrical breakdown strength. Expressing this linearly from the reference state (around $1 \mathrm{MPa}$ ) gives:

$$
E_{B D} / E_{B D, 0}=1-\frac{1}{4}\left(1-\frac{Y}{Y_{0}}\right)
$$

The linear relation can hold only for relatively small changes in Y, since Equation 28 states that the limiting value of the relative electrical breakdown strength $\left(E_{B D} / E_{B D, 0}\right)$ is 0.75 .

A logarithmic relation may be more applicable to describe the rule of thumb stated above (25\% decrease in electrical breakdown strength upon a ten-fold decrease in the Young's modulus):

$$
E_{B D} / E_{B D, 0}=1+\frac{1}{4} \log \frac{Y}{Y_{0}}
$$

This equation states that if the elastic modulus decreases by four decades, then electrical breakdown strength is zero. This seems a more realistic description, since silicone 
elastomers with elastic moduli of $100 \mathrm{~Pa}$ are not real elastomers with respect to mechanical properties, in the sense that they are hardly free-standing as thin films and will be very extensible with a large permanent set.

Using the logarithmic relation then produces the following expression for the figure of merit for the bimodal elastomer utilized as a generator:

$$
\frac{\epsilon_{r} E_{B D}^{2}}{\epsilon_{r, 0} E_{B D, 0}^{2}}=\left(1+\frac{1}{4} \log \frac{Y}{Y_{0}}\right)^{2}
$$

This relation is plotted in Figure 15. From the equation, it is obvious that these soft elastomers do not hold any promise in relation to being used as generator materials, since even the smallest decrease in elastic moduli leads to a reduction in the figure of merit. Of course, these calculations are based on rules of thumbs, but nevertheless, the interpretation gives a clear indication that softer elastomers do not solve challenges for all dielectric elastomer applications, and the interpretation (whether logarithmic or linear) of breakdown data does not influence the overall conclusion. Additionally, soft elastomers are more prone to failure as a result of electromechanical instability[118], which is a factor not accounted for in the figures of merit.

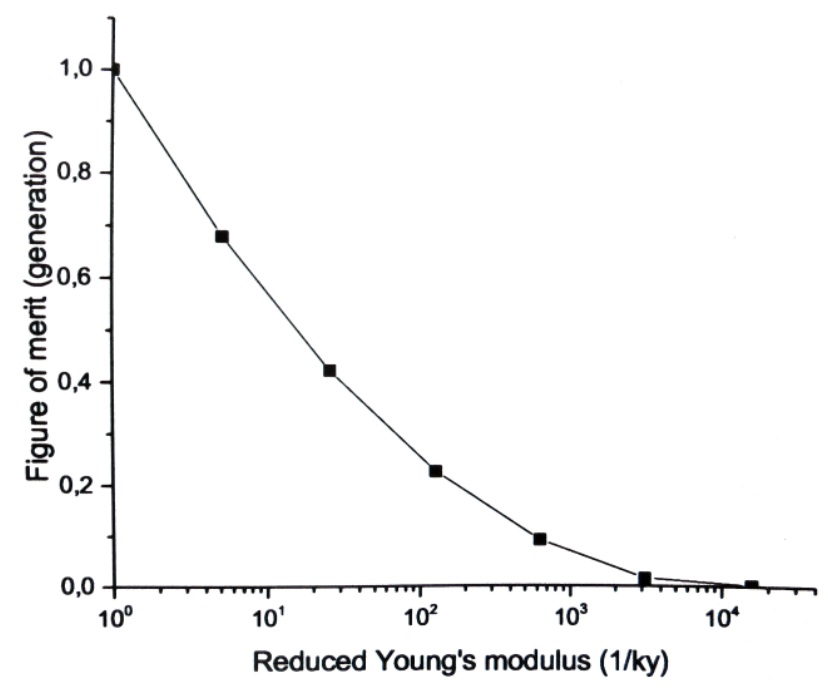

Figure 15: Illustration of the figure of merit for low-elastic modulus elastomers utilized as generators (Equation 31). 


\section{Comparison of the approaches}

In order to provide an indication of the applicability of the scaling laws derived earlier, this section presents achieved results from the literature compared to the scaling laws. In the following, we use Elastosil RT625 as the reference material, as it is a very typical silicone elastomer and is highly reliable. Data for the reference elastomer are shown in Table 3. Another commercially available elastomer often utilized for dielectric elastomers is Sylgard 186, produced by Dow. However, this elastomer is heavily filled with additives, which makes it prone to changing properties over time when applying a high voltage. Sylgard is nevertheless often used when the requirement of low elastomer premix viscosity is weighted the highest.

Table 3: A selection of the best results from the literature obtained to date. The figures of merit are introduced in Equations 4 and 5.

\begin{tabular}{|c|c|c|c|c|c|c|}
\hline Description & $\varepsilon_{\mathbf{r}}$ & $\begin{array}{l}\text { Y } \\
\text { [MPa] }\end{array}$ & $\begin{array}{l}\mathbf{E}_{\mathrm{B}} \\
{[\mathrm{V} / \mu \mathrm{m}]}\end{array}$ & $\begin{array}{l}\mathbf{F}_{\text {om_ }} / \mathbf{F}_{\text {om_ref }} \\
\text { (DEA) }\end{array}$ & $\begin{array}{l}\mathbf{F}_{\text {om_ }} / \mathbf{F}_{\text {om_ref }} \\
\text { (DEG) }\end{array}$ & Ref. \\
\hline $\begin{array}{l}\text { Reference } \\
\text { Elastosil RT625 }\end{array}$ & $\begin{array}{l}3 \\
@ 0.1 \mathrm{~Hz}\end{array}$ & 1 & 80 & 1 & 1 & [76] \\
\hline $\begin{array}{l}\text { Commercial } \\
\text { Sylgard } 186\end{array}$ & $\begin{array}{l}2.8 \\
@ 0.1 \mathrm{~Hz}\end{array}$ & 0.7 & 144 & 1.33 & 3.02 & [119] \\
\hline $\begin{array}{l}\text { TiO }_{2} \text {-filled } \\
\text { Sineskin A/B/C 50\% silicone + } \\
\left.\text { 30wt.\% } \mathrm{TiO}_{2} \text { (rutile, } 10 \mu \mathrm{m}\right)\end{array}$ & $\begin{array}{l}7.5 \\
\text { @ 10Hz }\end{array}$ & 0.016 & 10 & 156.3 & 0.04 & [120] \\
\hline $\begin{array}{l}\text { Various titanate-filled } \\
\mathrm{RT} 25+20 \mathrm{vol} \% \mathrm{BaTiO}_{3} \\
(<3 \mu \mathrm{m})\end{array}$ & $\begin{array}{l}7.5 \\
\text { @ } 1 \mathrm{kHz}\end{array}$ & 0.2 & 40 & 12.5 & 0.63 & [121] \\
\hline $\begin{array}{l}\text { Core-shell fillers } \\
\text { XLR630 + 6wt.\% } \mathrm{TiO}_{2} / \mathrm{SiO}_{2} \\
\text { Aeroxide (STX801) }\end{array}$ & $\begin{array}{l}3.88 \\
\text { @ } 1 \mathrm{MHz}\end{array}$ & 0.187 & 173 & 6.92 & 6.05 & [33] \\
\hline
\end{tabular}




\begin{tabular}{|c|c|c|c|c|c|c|}
\hline $\begin{array}{l}\text { Blends } \\
\text { BJB TC5005 + 40phr plasticiser }\end{array}$ & $\begin{array}{l}5.2 \\
@ 0.1 \mathrm{~Hz}\end{array}$ & 0.05 & 10 & 34.7 & 0.02 & [122] \\
\hline $\begin{array}{l}\text { Blends } \\
\text { MJK4/13 + 5wt.\% PEG-PDMS } \\
\text { block copolymer }\end{array}$ & $\begin{array}{l}4.7 \\
@ 0.1 \mathrm{~Hz}\end{array}$ & 0.123 & 103 & 12.7 & 2.43 & [96] \\
\hline $\begin{array}{l}\text { Blends } \\
\text { LR3043/50 + 30phr } \\
\text { chloropropyl-functional silicone } \\
\text { oil (LMS-152) }\end{array}$ & $\begin{array}{l}4.4 \\
@ 0.1 \mathrm{~Hz}\end{array}$ & 1.35 & 130 & 1.09 & 3.87 & [87] \\
\hline $\begin{array}{l}\text { Chemically modified } \\
\text { Synthesised azido-functional } \\
\text { siloxane copolymers OH-PDMS } \\
+ \text { 5.6wt.\% nitrobenzene }\end{array}$ & $\begin{array}{l}14.2 \\
@ 0.1 \mathrm{~Hz}\end{array}$ & 0.08 & 69 & 20.8 & 1.25 & [123] \\
\hline $\begin{array}{l}\text { Chemically modified } \\
\text { OH-poly-methylvinyl-siloxane } \\
+48 \text { wt.\% 3- } \\
\text { mercaptopropionitrile }\end{array}$ & $\begin{array}{l}10.1 \\
@ 0.1 \mathrm{~Hz}\end{array}$ & 0.15 & 11 & 22.4 & 0.24 & [124] \\
\hline $\begin{array}{l}\text { Chemically modified } \\
\text { Synthesized copolysiloxanes + } \\
\text { 10wt.\% chloropropyl-groups }\end{array}$ & $\begin{array}{l}4.9 \\
@ 0.1 \mathrm{~Hz}\end{array}$ & 1 & 94 & 1.63 & 2.18 & [92] \\
\hline
\end{tabular}

For traditional composites, two examples of excellent dielectric elastomers are shown in Table 3. RT625 + 20 vol\% $\mathrm{BaTiO}_{3}[121]$ has an improved figure of merit for actuation of 12.5, and the XLR $630+6 \mathrm{wt} \%$ core-shell $\mathrm{TiO}_{2}-\mathrm{SiO}_{2}$ system[33] possesses a figure of merit of 6 in terms of generation. The very high figure of merit (actuation) value is due mainly to a very low orientation parameter $\Phi$ (as defined in Equation 6) value, as verified by the very low Young's modulus of the composite. The reliability of this system, however, can be questioned, but the illustrated core-shell fillers have been modified to provide excellent interactions with the silicone polymer. Here, an improvement with respect to the figure of merit for actuation is limited, but due to increased electrical breakdown strength, the figure of 
merit for generation becomes large. This value is of course low compared to the predictions in Figure 6, but core-shell fillers make a limited contribution to dielectric permittivity, due to the compatibilizing but electrically insulating $\mathrm{SiO}_{2}$ shell layer. Realizing compatible $\mathrm{TiO}_{2}$ or other high-permittivity fillers without the insulating shell layer would be of great interest to the dielectric elastomer society.

As examples of blends with excellent figures of merit, the BJB TC5005 + 40phr system of so-called "plasticiser"[122] excels with a value of 34.7 for actuation. This composition will most likely phase-separate over time (even without the presence of an electrical field), due to the high concentration of incompatible oil (in which case, plasticizer is not the correct term). Of the more reliable elastomers, the PEG-PDMS system[96], with a figure of merit for actuation of 12.7, can be mentioned here, as this value agrees well with the prediction in Figure 10. Likewise, the figure of merit for generation of 2.4 agrees well with the prediction. As an example of another system, the realized figure of merit of chloropropyl oil[87] in silicone elastomers is higher than predicted in Figure 11, due to the voltage stabilization effect of chloropropyl. Equations 19 and 24 therefore seem to surface as realistic predictions of blend-based dielectric elastomer formulations.

For the chemically modified elastomer, figures of merit for actuations of 20.8 and 22.4, respectively, were achieved for two different systems, namely one with very high permittivity moiety in low concentrations[123] and one with relatively high permittivity moiety in relatively high concentrations[124]. Neither materials showed any significant promise for use in generators, due to decreased electrical breakdown strength. For these two material systems, Equations 25 and 26 do not hold, since the equations were derived under the assumption that grafting moiety does not affect electrical breakdown strength, i.e. in very low concentrations compared to the percolation threshold. One example of a system fulfilling this criterion is the chloropropyl functionalized (silicone copolymer) elastomer[92], with 
figures of merit of 1.63 and 2.18, respectively, for actuation and energy generation. These numbers agree far better with the predictions in Equations 25 and 26.

\section{Combinatorial approaches}

The above discussions show that each methodology has its shortcomings, either inherent in the resulting elastomer or during the processing thereof. Of course, one can claim that processing issues can always be overcome, but working with high-viscosity polymers is simply not easy. Processing is relatively uncomplicated for thermoplastics where a series of processes can be utilized. For elastomers, however, this is not possible, due to the excessive heat developed from mixing, thus accelerating the curing speed and thereby increasing viscosity even further.

As can be seen from Table 1, the major issue in compositing methodology is processing, as well as the Mullins effect over time. This effect can be avoided by softening the particle-particle interaction, e.g. by adding oil to the composites. Yet another obvious feature of this combined approach is that the viscosity of the otherwise very viscous composite reaction mixture will decrease and the need for solvent reduce, thereby easing processing. This approach was the idea behind article[98], in which chloropropyl functional oil was added to $\mathrm{TiO}_{2}$-based composite. Very interestingly, the synergistic effects were favorable and led to expected results with respect to both elastic and dielectric properties, except that the dielectric loss of the composite actually decreased following the addition of chloropropyl functional silicone oil. After some time stretching, equilibration in the cured elastomer was observed, leading to even more favorable properties - explained by the oil migrating to the particle interface and thus altering homogeneity on the nano-scale, albeit still with no effect on micro-scale homogeneity. Increases in relative dielectric permittivity were observed as well as elastomer softening, which was ensured not to stem from volatile evaporation or other ageing phenomena. Of course, this initial time-dependency of the 
properties complicates processing if the elastomers equilibrate after curing on a temperaturedependent timescale, but on the other hand, it gives a clear indication that control of the network/composite structure on the nano-scale is open to improved or even completely novel properties. For now, this notion remains unexplored, due to the complexity of determining silicone compositions on the nano-scale.

Another obvious combinational approach is to couple grafted voltage stabilizing moieties to the elastomer matrix of all elastomer compositions with an increased ratio of dielectric permittivity over the Young's modulus, thereby gaining further electromechanical stability. This is most likely the approach of the novel Wacker film, as discussed earlier.

Additionally, with increasing knowledge on the fundamentals of physical and electrical phenomena in actuating dielectric elastomers, more combinatorial approaches will probably allow silicone-based dielectric elastomers to pave their way into products based on dielectric elastomer transducers. Furthermore, completely novel operational principles such as ion-implanted electrodes[125], pulsed actuation[126], and various skeletal structures utilized to support the films, just to mention a few, may allow for different elastomer requirements and thus open up an opportunity for new materials, which, for example, are currently limited by their relatively high conductivity or high losses.

Pre-stretching is another obvious method to increase actuation, as originally proven by Pelrine et al.[24] and Kornbluh et al.[127] However, pre-stretching imposes some restrictions on the design of the dielectric elastomer transducer, since rigid, heavy frames etc. are required and thus destroy some of the most favorable features of dielectric elastomers[128]. However, on the other hand, pre-stretching eliminates some failure modes[129-131] and enhances electrical breakdown strength significantly[132,133]. This is not discussed further within this review, since the method usually causes some long-term relaxation of the silicone elastomers (loss of tension). Therefore, in the current state, it is not 
regarded as a means of producing reliable silicone-based dielectric elastomers. Small prestretches, performed within the linear viscoelastic regime, are of course acceptable and may lead to incremental improvements. However, the major improvements reported in the literature[134-136] have been observed above the 50\% strain, and this is seen as unfeasible from a product reliability perspective .

\section{Interactions of dielectric elastomer films with the environment}

A topic almost ignored in this review is on the subject of dielectric elastomer transducer electrodes. For all calculations and derivations, it is assumed that the electrodes are compliant, i.e. that they do not contribute with any significant stiffness to the dielectric elastomer, and that their conductivity is so great that they supply the voltage almost instantaneously (at least on a timescale of microseconds[13]).

In the context of elastomer blends, it was discussed how incompatible oils may migrate to the electrodes over time. However, the opposite situation, i.e. oils from the electrodes migrating to the elastomer, may also occur if, for example, the electrode systems of conductive fillers diluted in silicone oil are utilized. Such migration is thermodynamically favorable and leads to the dielectric elastomer swelling, i.e. an increase in volume. Furthermore, swelling softens the elastomer and electrical breakdown strength decreases as discussed in the back to "Chapter 3". Since the volume of the electrodes is usually small compared to the volume of the dielectric elastomer itself, this effect is not supposed to pose any threat to the elastomer. However, the electrode may dry out when silicone oil migrates to the elastomer, since conductive — usually carbon-based — compounds have no driving force for entering the silicone elastomer force. A dry electrode may then lead to the elastomer tearing as well as loss of conductivity if particle-particle connectivity ceases.

It is therefore highly recommended, when formulating electrode materials for dielectric elastomers, that the electrode matrix is not soluble in dielectric elastomers. 
As a final note on the interactions of dielectric elastomers with their surroundings, it is also important to take precautions if formulating with polar and hygroscopic materials, since water uptake in elastomers may alter their electrical properties significantly[137] towards what appear to be improvements as a result of increased dielectric permittivity; in addition, it also paves the way towards slightly conducting materials. Silicone elastomers are highly permeable[90], and therefore it is unavoidable to have water transport in the films, for example, if a driving force for this is present. In general, silicone elastomers are very resistant to most environments, but the improvement strategies proposed within this review may alter transport properties, in which case further testing may be required.

\section{Conclusions}

Within this review it has been shown how certain optimization methods excel for some applications of dielectric elastomer transducers whereas other optimization methods solve yet other challenges. In general, dielectric elastomers from compositing with metal oxides are only suitable for generators but are by far the most suitable type of elastomer for this application. The other discussed methods result in relatively softer elastomers and are thus more suited for actuators. In general, all discussed optimization methods hold significant promise for one or more operational mode of the dielectric elastomer transducer, and especially when combined pairwise or in even more complex combinations. As an example, high permittivity moieties may be grafted to the silicone elastomer backbone in order to follow for better miscibility of likewise functionalized silicone oil. Thereby a soft and high permittivity elastomer is achieved. This resulting elastomer can be further optimized towards becoming more electrical resistant by e.g. addition of minute amounts of voltage stabilization compounds such as $\mathrm{ZnO}$ or alternatively by a larger amount of reinforcing fillers to give a relatively harder elastomer with very high electrical breakdown strength. In this manner, many new types of elastomers are believed to appear in the near future with the increased 
knowledge of the effect of certain moieties on the electro-mechanical properties.

Nonetheless, in order to discover fully the potential of dielectric elastomers more focus should be directed towards the structural configuration of high permittivity moieties and the influence thereof on the electro-mechanical properties. As an example no coherent knowledge has been established on how electrical breakdown takes place in these soft materials. Recently, the voltage-stabilization of elastomers has been explored and demonstrated, but in order to understand fully the phenomena behind this, knowledge of the elastomers on the nanoscale is required. The nanoscopic/microscopic phase separation is believed to be significant for most silicone elastomers simply because silicone is immiscible with most substances, and therefore reported lack of macroscopic phase separation is no guarantee for lack of microscopic or nanoscopic phase separation. Therefore it is believed that further knowledge on this scale is the way towards understanding the phenomena leading to electro-mechanical failure and thus subsequently be able to use controlled phase separation as means of optimizing silicone-based dielectric elastomers.

\section{References}

[1] P. Brochu, Q. Pei, Macromol. Rapid Commun. 2010, 31, 10.

[2] C. Löwe, X. Zhang, G. Kovacs, Adv. Eng. Mater. 2005, 7, 361.

[3] S. Bauer, S. Bauer-Gogonea, I. Graz, M. Kaltenbrunner, C. Keplinger, R. Schwödiauer, Adv. Mater. 2014, 26, 149.

[4] D. Chen, Q. Pei, Chem. Rev. 2017, 117, 11239.

[5] I. A. Anderson, T. Gisby, T. McKay, B. M. O'Brien, E. P. Calius, J. Appl. Phys. 2012, $112,41101$.

[6] S. Rosset, H. R. Shea, Appl. Phys. Rev. 2016, 3, 31105.

[7] R. Pelrine, R. D. Kornbluh, Q. Pei, J. Joseph, Science 2000, 287, 836. 
[8] F. Habrard, J. Patscheider, G. Kovacs, Adv. Eng. Mater. 2014, 16, 1133.

[9] M. Benslimane, P. Gravesen, P. Sommer-Larsen, Proc. SPIE 2002, 4695, 150.

[10] B. Fasolt, M. Hodgins, G. Rizzello, S. Seelecke, Sens. Actuators A Phys. 2017, 265, 10.

[11] M. F. Appel, W. E. van der Veer, T. Benter, J. Am. Soc. Mass. Spectrom. 2002, 13, 1170.

[12] B. Matthews, J. Li, S. Sunshine, L. Lerner, J. W. Judy, IEEE Sens. J. 2002, 2, 160.

[13] S. Rosset, H. R. Shea, Appl. Phys. A Mater. Sci. Process. 2013, 110, 281.

[14] D. McCoul, W. Hu, M. Gao, V. Mehta, Q. Pei, Adv. Elect. Mater. 2016, 2, 1500407.

[15] W. W. Vogt, R. D. Evans, Ind. Eng. Chem. 1923, 15, 1015.

[16] S. Shian, K. Bertoldi, D. R. Clarke, Adv. Mater. 2015, 27, 6814.

[17] M. Benslimane, P. Gravesen, P. Sommer-Larsen, Proc. SPIE 2002, 4695, 150.

[18] R. Kornbluh, R. Pelrine, Q. Pei, R. Heydt, S. Stanford, S. Oh, J. Eckerle, Proc. SPIE 2002, 4698, 254.

[19] R. Pelrine, R. Kornbluh, G. Kofod, Adv. Mater. 2000, 12, 1223.

[20] http://www.stretchsense.com/

[21] T. G. McKay, B. M. O’Brien, E. P. Calius, I. A. Anderson, Appl. Phys. Lett. 2011, 98, 142903.

[22] C. Jean-Mistral, S. Basrour, J.-J. Chaillout, Smart Mater. Struct. 2010, 19, 085012.

[23] R. Pelrine, R. Kornbluh, J. Eckerle, P. Jeuck, S. Oh, Q. Pei, S. Stanford, Proc. SPIE 2001, 4329, 148.

[24] R. E. Pelrine, R. D. Kornbluh, J. P. Joseph, Sens. Actuators A Phys. 1998, 64, 77.

[25] X. Zhao, W. Hong, Z. Suo, Phys. Rev. B 2007, 76, 134113.

[26] P. Sommer-Larsen, J. Hooker, G. Kofod, K. West, M. Benslimane, P. Gravesen, Proc. SPIE 2001, 4329, 157.

[27] P. Sommer-Larsen, A. L. Larsen, Proc. SPIE 2004, 5385, 68. 
[28] T. G. McKay, E. Calius, I. A. Anderson, Proc. SPIE 2009, 7287, 72870P-1.

[29] F. B. Madsen, A. E. Daugaard, S. Hvilsted, A. L. Skov, Macromol. Rapid Commun. 2016, 37, 378 .

[30] M. A. Brook, Silicon in Organic, Organometallic, and Polymer Chemistry, John Wiley \& Sons, New York, United States, 2000.

[31] B. Kussmaul, S. Risse, G. Kofod, R. Wache, M. Wegener, D. N. McCarthy, H. Krüger, R. Gerhard, Adv. Funct. Mater. 2011, 21, 4589.

[32] E. Delebecq, N. Hermeline, A. Flers, F. Ganachaud, ACS Appl. Mater. Interfaces 2012, 4,3353 .

[33] S. Vudayagiri, S. Zakaria, L. Yu, S. S. Hassouneh, M. Benslimane, A. L. Skov, Smart Mater. Struct. 2014, 23, 105017.

[34] M. Cazacu, M. Ignat, C. Racles, M. Cristea, V. Musteata, D. Ovezea, D. Lipcinski, J. Compos. Mater. 2014, 48, 1533.

[35] H. Zhao, L. Zhang, M. Yang, Z. Dang, J. Bai, Appl. Phys. Lett. 2015, 106, 092904.

[36] G. Gallone, F. Carpi, D. De Rossi, G. Levita, A. Marchetti, Mater. Sci. Eng. C 2007, 27, 110.

[37] D. Yang, L. Zhang, H. Liu, Y. Dong, Y. Yu, M. Tian, J. Appl. Polym. Sci. 2012, 125, 2196.

[38] I. Babu, G. de With, Compos. Sci. Technol. 2014, 91, 91.

[39] M. Razzaghi-Kashani, N. Gharavi, S. Javadi, Smart Mater. Struct. 2008, 17, 065035.

[40] Z. M. Dang, B. Xia, S. H. Yao, M. J. Jiang, H. T. Song, L. Q. Zhang, D. Xie, Appl. Phys. Lett. 2009, 94, 042902.

[41] K. Goswami, A. E. Daugaard, A. L. Skov, RSC Adv. 2015, 5, 12792.

[42] S. S. Hassouneh, A. E. Daugaard, A. L. Skov, Macromol. Mater. Eng. 2015, 300, 542. 
[43] M. Iacob, G. Stiubianu, C. Tugui, L. Ursu, M. Ignat, C. Turta, M. Cazacu, RSC Adv. 2015, 5, 45439 .

[44] J. E. Q. Quinsaat, M. Alexandru, F. A. Nüesch, H. Hofmann, A. Borqschulte, D. M.

Opris, J. Mater. Chem. A 2015, 3, 14675.

[45] J. Diani, B. Fayolle, P. Gilormini, Eur. Polym. J. 2009, 45, 601.

[46] S. Cantournet, R. Desmorat, J. Besson. Int. J. Solids Struct. 2009, 46, 2255.

[47] S. Zakaria, L. Yu, G. Kofod, A. L. Skov, Mater. Today Commun. 2015, 4, 204.

[48] J. Fröhlich, W. Niedermeier, H. D. Luginsland, Compos. Part. A Appl. Sci. Manuf. 2005, $36,449$.

[49] A. D. Drozdov, A. Dorfmann, Polym. Eng. Sci. 2002, 42, 591.

[50] R. Yang, Y. Song, Q. Zheng, Polymer 2017, 116, 304.

[51] A. Kausar, Fuller. Nanotub. Car. N. 2017, 25, 109.

[52] D. Ogawa, K. Nakamura, Jpn. J. Appl. Phys. 2016, 55, 01 AE22.

[53] C. Chevigny, N. Jouault, F. Dalmas, F. Boué, J. Jestin, J. Polym. Sci. Part B Polym. Phys. 2011, 49, 781.

[54] S. Babu, K. Singh, A. Govindan, Appl. Phys. A 2012, 107, 697.

[55] L. Yu, A. L. Skov, RSC Adv. 2017, 7, 45784.

[56] L. Yu, A. L. Skov, Int. J. Smart Nano Mater. 2016, 6, 268.

[57] M. Kollosche, H. Stoyanov, H. Ragusch, S. Risse, A. Becker, G. Kofod, 2010 10th IEEE Int. Conf. Solid Dielectr. 2010, 1.

[58] P. Brochu, H. Stoyanov, X. Niu, Q. Pei, Smart Mater. Struct. 2013, 22, 055022.

[59] A. L. Skov, unpublished data by the author.

[60] M. A. Brook, H. U. Saier, J. Schnabel, K. Town, M. Maloney, Ind. Eng. Chem. Res. 2007, 46, 8796 
[61] P. Lotz, M. Matysek, P. Lechner, M. Hamann, H. F. Schlaak, Proc. SPIE 2008, 6927, 692723-1.

[62] S. Vudayagiri, L. Yu, S. S. Hassouneh, U. Hansen, A. L. Skov, Polym. Plast. Technol. Eng. 2015, 54, 425.

[63] L. A. Dissado, J. C. Fothergill, Electrical Degradation and Breakdown in Polymer, Technology \& Engineering, Peter Peregrinus Ltd., London, United Kingdom, 1992.

[64] P. Mazurek, W. Wirges, R. Gerhard, A. L. Skov, J. Appl. Polym. Sci. 2016, 113, 44153.

[65] P. Mazurek, S. Hvilsted, A. L. Skov, Polymer 2016, 87, 1.

[66] P. Mazurek, A. E. Daugaard, M. Skolimowski, S. Hvilsted, A. L. Skov, RSC Adv. 2015, $5,15379$.

[67] S. Hunt, T. G. McKay, I. A. Anderson, Appl. Phys. Lett. 2014, 104, 113701.

[68] F. Carpi, G. Gallone, F. Galantini, D. De Rossi, Adv. Funct. Mater. 2008, 18, 235.

[69] A. L. Larsen, P. Sommer-Larsen, O. Hassager, Proc. SPIE 2004, 5385, 108.

[70] F. B. Madsen, L. Yu, A. E. Daugaard, S. Hvilsted, A. L. Skov, Proc. SPIE 2015, 9430, 943012-1.

[71] M. Dascalu, S. J. Dünki, J. Q. Quinsaat, Y. S. Ko, D. M. Opris, $R S C A d v .2015,5,104516$.

[72] A. H. A Razak, L. Yu, A. L. Skov, $R S C A d v$. 2017, 7, 17848.

[73] B. Kussmaul, S. Risse, G. Kofod, R. Wache, M. Wegener, D. N. McCarthy, H. Kruger, R. Gerhard, Adv. Funct. Mater. 2011, 21, 4589.

[74] F. B. Madsen, S. B. Zakaria, A. L. Skov, Polym. Plast. Technol. Eng. 2017, 56, 83.

[75] F. B. Madsen, S. B. Zakaria, L. Yu, A. L. Skov, Adv. Eng. Mater. 2016, 18, 1154.

[76] L. Yu, F. B. Madsen, A. L. Skov, Int. J. Smart Nano Mater., DOI: 10.1080/19475411.2017.1376358.

[77] J. Neuefeind, A. L. Skov, J. E. Daniels, V. Honki, RSC Adv. 2016, 6, 95910.

[78] S. Vudayagiri, M. D. Junker, A. L. Skov, Polym. J. 2013, 45, 871. 
[79] S. Vudayagiri, A. L. Skov, Polym. Adv. Tech. 2014, 25, 249.

[80] S. Vudayagiri, PhD thesis: Large-scale processing of dielectric electroactive polymers, Technical University of Denmark, 2014.

[81] J. Meyer, C. A. Smith, Ipc Apex Expo 2012, 3, 1622.

[82] F. B. Madsen, I. Javakhishvili, R. E. Jensen, A. E. Daugaard, S. Hvilsted, A. L. Skov, Polym. Chem. 2014, 5, 7054.

[83] C. Tugui, A. Bele, V. Tiron, E. Hamciuc, C. D. Varganici, M. Cazacu, J. Mater. Chem. C 2017, 5, 824 .

[84] S. J. Dünki, F. A. Nüesch, D. M. Opris, J. Mater. Chem. C 2016, 4, 10545.

[85] A. Bele, M. Cazacu, C. Racles, G. Stiubianu, D. Ovezea, M. Ignat, Adv. Eng. Mater. 2015, 17, 1302.

[86] F. B. Madsen, I. Dimitrov, A. E. Daugaard, S. Hvilsted, A. L. Skov, Polym. Chem. 2013, $4,1700$.

[87] F. B. Madsen, L. Yu, S. Hvilsted, A. L. Skov, Proc. SPIE 2015, 9430, 94301D-1.

[88] J. Morgan, T. Chen, R. Hayes, T. Dickie, T. Urlich, M. A. Brook, Polym. Chem. 2017, 8, 2743.

[89] F. Ganachaud, S. Boileau, B. Boury, Silicon Based Polymers: Advances in Synthesis and Supramolecular Organization, Springer Science \& Business Media, London, United Kingdom, 2008.

[90] J. Zhang, S. Liang, L. Yu, A. L. Skov, H. M. Etmimi, P. E. Mallon, A. Adronov, M. A. Brook, J. Polym. Sci. A: Polym. Chem. 2016, 54, 2379.

[91] M. Pascoal, M. A. Brook, F. Gonzaga, L. Zepeda-Velazquez, Eur. Polym. J. 2015, 69, 429.

[92] F. B. Madsen, L. Yu, A. E. Daugaard, S. Hvilsted, A. L. Skov, RSC Adv. 2015, 5, 10254. 
[93] V. Englund, R. Huuva, S. M. Gubanski, T. Hjertberg, Polym. Degrad. Stab. 2009, 94, 823.

[94] Y. Yamano, IEEE Trans. Dielectr. Electr. Insul. 2006, 13, 773.

[95] Y. Yamano, H. Endoh, IEEE Trans. Dielectr. Electr. Insul. 1998, 5, 270.

[96] A. H. A Razak, P. Szabo, A. L. Skov, RSC Adv. 2015, 5, 53054.

[97] A. H. A Razak, A. L. Skov, RSC Adv. 2017, 7, 468.

[98] F. B. Madsen, L. Yu, P. Mazurek, A. L. Skov, Smart Mater. Struct. 2016, 25, 075018.

[99] R. V. Mateiu, L. Yu, A. L. Skov, Proc. SPIE 2017, 10163, 1016328-1.

[100] A. E. Daugaard, S. S. Hassouneh, M. N. Mazurek, A. G. Bejenariu, A. L. Skov, Proc. SPIE 2013, 8687, 868729-1.

[101] S. S. Hassouneh, L. Yu, A. L. Skov, A. E. Daugaard, J. Appl. Polym. Sci. 2017, 134, 44767.

[102] Gelest, Inc., Silicon Compounds: Silanes and Silicones: a Survey of Properties and Chemistry, Gelest, Incorporated, United States, 2004.

[103] N. L. Larry, S. Judith, G. Yan, E. Robert, Platinum Metals Rev. 1997,41, 66.

[104] R. Heydt, R. Kornbluh, J. Eckerle, R. Pelrine, Proc. SPIE 2006, 6168, 61681M-1.

[105] R. Heydt, R. Pelrine, J. Joseph, J. Eckerle, R. Kornbluh. J Acoust Soc Am. 2000, 107, 833.

[106] T. Hou, Y. Yang, H. Zhang, J. Chen, L. Chen, Z. Wang, Nano Energy 2013, 2, 856.

[107] Y. Yang, H. Zhang, Y. Liu, Z. Lin, S. Lee, Z. Lin, C. Wong, Z. Wang, ACS Nano 2013, 7, 2808 .

[108] S. B. Zakaria, P. H. F. Morshuis, B. M. Yahia, K. Gernaey, A. L. Skov, Proc. SPIE 2014, 9056, 90562V-1.

[109] D. Gatti, H. Haus, M. Matysek, B. Frohnapfel, C. Tropea, H. F. Schlaak, Appl. Phys. Lett. 2014, 104, 052905. 
[110] A. L. Larsen, K. Hansen, P. Sommer-Larsen, O. Hassager, A. Bach, S. Ndoni, M. Jørgensen, Macromolecules 2003, 36, 10063.

[111] S. M. G. Frankær, M. K. Jensen, A. G. Bejenariu, A. L. Skov, Rheol. Acta 2012, 51, 559.

[112] A. V. Marcelo, A. B. Miguel, M. V. Enrique, Macromolecules 1996, 29, 4072.

[113] K. Urayama. Polym. J. 2008, 40, 669.

[114] F. B. Madsen, A. E. Daugaard, C. Fleury, S. Hvilsted, A. L. Skov, RSC Adv. 2014, 4, 6939.

[115] M. K. Jensen, H. K. Rasmussen, A. L. Skov, O. Hassager, Rheol. Acta 2011, 50, 729.

[116] L. Cai, T. E. Kodger, R. E. Guerra, A. F. Pegoraro, M. Rubinstein, D. A. Weitz, Adv. Mater. 2015, 27, 5132.

[117] M. Vatankhah-Varnoosfaderani, W. F. M. Daniel, A. P. Zhushma, Q. Li, B. J. Morgan, K. Matyjaszewski, D. P. Armstrong, R. J. Spontak, A. V. Dobrynin, S. S. Sheiko, Adv. Mater. 2017, 29, 1604209.

[118] C. Keplinger, T. Li, R. Baumgartner, Z. Suo, S. Bauer, Soft Matter 2012, 8, 285.

[119] R. Pelrine, R. Kornbluh, J. Joseph, R. Heydt, Q. Pei, S. Chiba, Mater. Sci. Eng. C 2000, $11,89$.

[120] F. Carpi, D. De Rossi, IEEE Trans. Dielectr. Electr. Insul. 2005, 12, 835.

[121] H. Böse, D. Uhl, K. Flittner, H. Schlaak, Proc. SPIE 2011, 7976, 79762J-1.

[122] F. Galantini, F. Carpi, G. Gallone, Smart Mater. Struct. 2013, 22, 104020.

[123] F. B. Madsen, L. Yu, A. E. Daugaard, S. Hvilsted, A. L. Skov, Polymer 2014, 55, 6212.

[124] S. J. Dünki, Y. S. Ko, F. A. Nüesch, D. M. Opris, Adv. Funct. Mater. 2015, 25, 2467.

[125] S. Rosset, M. Niklaus, P. Dubois, H. R. Shea, J. Microelectromech. S. 2009, 18, 1300.

[126] T. Kobayashi, S. K. Smoukov, Int. J. Smart Nano Mater. 2014, 5, 217. 
[127] R. D. Kornbluh, R. Pelrine, J. Joseph, R. Heydt, Q. Pei, S. Chiba, Proc. SPIE 1999, $3669,149$.

[128] M. Duduta, R. J. Wood, D. R. Clarke, Adv. Mater. 2016, 28, 8058.

[129] J. Zhou, W. Hong, X. Zhao, Z. Zhang, Z. Suo, Int. J. Solids Struct. 2008, 45, 3739.

[130] M. Moscardo, X. Zhao, Z. Suo, Y. Lapusta, J. Appl. Phys. 2008, 104, 093503.

[131] J. Plante, S. Dubowsky. Int. J. Solids Struct. 2006, 43, 7727.

[132] S. Zakaria, P. H. F. Morshuis, M. Y. Benslimane, L. Yu, A. L. Skov, Smart Mater. Struct. 2015, 24, 55009.

[133] A. Tröls, A. Kogler, R. Baumgartner, R. Kaltseis, C. Keplinger, R. Schwödiauer, I. Graz, S. Bauer, Smart Mater. Struct. 2013, 22, 104012.

[134] D. McCoul, S, Rosset, N. Besse, H. Shea, Smart Mater. Struct. 2017, 26, 025015.

[135] J. Liang, L. Li, D. Chen, T. Hajagos, Z. Ren, S. Chou, W. Hu, Q. Pei, Nat. Commun. 2015, $6,8647$.

[136] Y. Cao, T. G. Morrissey, E. Acome, S. I. Allec, B. M. Wong, C. Keplinger, C. Wang, Adv. Mater. 2017, 29, 1605099.

[137] A. L. Skov, unpublished data by the author. 